\title{
Wachstum? Wohlstand und Lebensqualität! Möglichkeiten und Hürden der Verankerung sozial und ökologisch nachhaltiger Wirtschafts- und Gesellschaftspolitik in Österreich
}

\begin{abstract}
Markus Griesser ${ }^{\star}$ und Ulrich Brand ${ }^{* *}$
Zusammenfassung

In jüngster Zeit wurde auf internationaler Ebene ebenso wie in verschiedenen Ländern eine Vielzahl alternativer Indikatoren gesellschaftlichen Wohlstands und Fortschritts entwickelt. Den Ausgangspunkt bildete eine Kritik des Bruttoinlandsprodukts (BIP) als vermeintlich aussagekräftiger Wohlstandsindikator. Alternative Indikatoren sollen dabei Fortschritte und Probleme der Realisierung sozialer und ökologischer Nachhaltigkeit sowie unterschiedliche Dimensionen von Lebensqualität abbilden. Entsprechend können sie als Einsatzpunkte für ein sozial-ökologisch erweitertes Wohlstandsverständnis bzw. für eine lebensqualitäts- und wohlstandsorientierte Politik betrachtet werden, mit der die Orientierung an Wachstum und Wettbewerbsfähigkeit infrage gestellt werden kann. Doch obschon zahlreiche (Kollektiv-)Akteure über solche Kennzahlen verfügen, ist ihr politischer Impact bislang beschränkt. Mittels einer interpretativen Policy-Analyse geht es in diesem Beitrag um strategische Möglichkeiten einer besseren institutionellen Verankerung sozial und ökologisch nachhaltiger Wirtschafts- und Gesellschaftspolitik in Österreich.
\end{abstract}

Schlagwörter: Wohlstandsindikatoren, Kritik am BIP, alternative Wirtschafts- und Gesellschaftspolitik, lebensqualitäts- und wohlstandsorientierte Politik, interpretative Policy-Analyse

\section{Growth? Well-being and quality of life! Opportunities and barriers to improve the institutional foundation of sustainable policies in Austria}

\section{Abstract}

In recent years, different sets of alternative indicators of societal wealth and progress have been developed by political actors on an international level, but also in different nation states. The point of departure of these projects is a critique of GDP and the effort to replace or to supplement it by (additional) indicators mainly concerned with social and ecological sustainability. Therefore, they can be conceived as entry points for a broader understanding of societal well-being and thus for quality of life and well-being oriented forms of policy-making. But even though numerous actors nowadays are providing such indicators, their political impact is rather limited. Hence, from the perspective of an interpretive policy analysis the article is asking for the strategic opportunities to improve the institutional foundation of sustainable policies in Austria.

Keywords: Indicators of well-being, critique of GDP, alternative economic and social policies, quality of life and well-being oriented policies, interpretive policy analysis

\footnotetext{
* Markus Griesser, Hauptansprechpartner, Institut für Politikwissenschaft, Universität Wien. E-Mail: markus.griesser@ univie.ac.at

**Ulrich Brand, Institut für Politikwissenschaft, Universität Wien.E-Mail: ulrich.brand@univie.ac.at.

Dieser Artikel basiert auf Forschungen, die von Februar bis Oktober 2016 am Institut für Politikwissenschaft der Universität Wien durchgeführt und von der AK Wien finanziell gefördert wurden. Unser Dank gilt insbesondere Florian Wukovitsch und Georg Feigl von der AK Wien sowie den beiden anonymen GutachterInnen von Momentum Quaterly für produktive Kritik und viele Anregungen, die größtenteils berücksichtigt wurden.
} 


\section{Einleitung}

Seit Ende der 20ooer-Jahre intensiviert sich die Debatte über eine einseitig wachstumsorientierte Wirtschaftspolitik unter Ausklammerung sozialer und ökologischer Dimensionen. Vor diesem Hintergrund gewinnt auch die Problematisierung traditioneller Kennzahlen gesellschaftlichen Wohlstands und Fortschritts sowie die Forderung nach ihrer Ersetzung bzw. Ergänzung durch alternative Indikatoren (erneut) an Bedeutung. Im Zentrum der Auseinandersetzung steht das Bruttoinlandsprodukt (BIP) als jener Indikator, der ausgehend von den 1930er-Jahren zur „mächtigste[n] Kennzahl der Menschheitsgeschichte“ (Lepenies 2013: 9) avancierte (vgl. exemplarisch ebd.; Fioramonti 2013; Schmelzer 2016).

Wenngleich nicht durch sie in Gang gesetzt, erhielt die Debatte durch den Ausbruch der Finanzund Wirtschaftskrise ab 2007 zusätzlichen Antrieb. Exemplarisch zum Ausdruck kommt dies im Postulat der Europäischen Kommission (2013: 4), demzufolge „[t]he need for additional indicators [...] has been reinforced by the crisis“. Wie in der Wachstumsdebatte als solcher kommt so auch in der eng mit ihr verbundenen Indikatorendebatte - obschon häufig in indirekter und vermittelter Form - ein Bewusstsein der aktuellen Krise im Sinne eines Unbehagens darüber zum Ausdruck, was eigentlich Wohlstand und Lebensqualität bedeuten (vgl. exemplarisch Brangsch 2011; Jackson 2013). Schließlich ist die Krise nicht nur eine von Finanzmärkten und Wirtschaft, sondern sie artikuliert sich mit der ökologischen Krise sowie mit einer zunehmenden Krise sozialer Reproduktion und gesellschaftlicher Repräsentation (vgl. exemplarisch Brand/Wissen 2017: 21ff.; Demirović et al. 2011; Skidelsky/Skidelsky 2014).

Wie selbst die Europäische Kommission (2014: 7) mit Blick auf die sozialen und ökologischen Folgen der Krise betont, sei durch diese deutlich geworden, dass „eine Rückkehr zum Wachstumsmodell des vergangenen Jahrzehnts nicht nur illusorisch, sondern auch schädlich“ ist. Vordergründig markiert sie in den Augen verschiedener Akteure entsprechend eine Chance für die Überwindung einer einseitig am BIP-Wachstum orientierten Wirtschaftspolitik (vgl. etwa Europäische Kommission 2009: 3; OECD 2011: 14; Deutscher Bundestag 2013: v.a. 232ff.).

In den vergangenen Jahren wurden daher zahlreiche Vorschläge entwickelt, um das BIP durch alternative Indikatoren zu ersetzen bzw. zu ergänzen (für einen knappen Überblick vgl. Diefenbacher/Zieschank 2011; Bache/Reardon 2016: 44ff.). Dadurch geschärft wurde auch das Bewusstsein von der Unzulänglichkeit traditioneller Kennzahlen gesellschaftlichen Wohlstands und Fortschritts. Entsprechend konstatierte etwa das Europäische Parlament (2011: 2) in einer Resolution, dass "the need to improve data and indicators to complement GDP for overall societal development is increasingly recognised“. Dieses Problembewusstsein hat bislang jedoch kaum dazu beigetragen, den politischen Impact der Indikatorendebatte zu erhöhen (vgl. etwa Bache 2013: 31; Bleys/Whitby 2015: 164).

In den vergangenen Jahren rückten deshalb vereinzelt Fragen nach den wesentlichen Barrieren für den politischen Impact alternativer Indikatoren bzw. nach Möglichkeiten ihrer Überwindung in den Fokus der wissenschaftlichen Forschung (vgl. etwa Whitby et al. 2014; Bache/Reardon 2013, 2016). Und auch auf politischer Ebene sah etwa die Europäische Kommission (2013: 7) in ihrem Zwischenresümee zu der von ihr ab 2009 verfolgten GDP-and-Beyond-Initiative hier eine zentrale Herausforderung:

„With the novel ,GDP and Beyond' indicators

becoming increasingly available, the challenge now is how to link the new insights they provide with the existing tools for policy assessment and evaluation, be they econometric models or political narratives. Further attention also needs to be given to how to effectively integrate and communicate the available indicators and summarise data."

Eine Möglichkeit, ausgehend von der Debatte um alternative Kennzahlen gesellschaftlichen Fortschritts und Wohlstands, die institutionelle Verankerung lebensqualitäts- und wohlstandsorientierter Politik zu stärken, wird dabei in der Entwicklung eines neuen wirtschaftspolitischen Regulierungs- und Steuerungsrahmens gesehen. Dieser soll (im Sinne eines alternativen ,magischen Vier-' bzw. ,Vielecks der Wirtschaftspolitik') eine Reihe übergreifender Ziele umfassen, deren praktische Umsetzung durch spezielle Institutionen (z.B. Wohlstandsrat) zu gewährleisten, anhand eines Sets von Indikatoren zu messen und mithilfe eines darauf bezogenen Berichts- und Kontrollwesens zu überwachen wären (vgl. etwa Dullien/Van Treeck 2012; Feigl 2017).

Der vorliegende Beitrag tritt gegenüber solchen Vorschlägen einen Schritt zurück und fragt aus politikwissenschaftlicher Perspektive nach zentralen Barrieren für eine institutionelle Verankerung lebensqualitäts- und wohlstandsorientierter Politik in Österreich sowie nach den strategischen Möglichkeiten 
ihrer Überwindung. ${ }^{1}$ Entscheidend für Lebensqualität sind dabei die individuellen und gesellschaftlichen materiellen wie immateriellen Bedingungen, d.h. Einkommen und Vermögen, (Aus-)Bildungsmöglichkeiten, Berufschancen bzw. Zugang zu und Qualität von Erwerbsarbeit, öffentliche Daseinsvorsorge und soziale Sicherung, Gesundheitsvorsorge und Lebenserwartung, wirtschaftliche und soziale Stabilität, Meinungsfreiheit und politische Beteiligungsmöglichkeiten sowie eine lebenswerte Umwelt. Wichtig ist aber auch, inwiefern diese Bedingungen zur Steigerung der Lebensqualität genutzt werden können, d.h. die Menschen die Befähigung dazu haben (vgl. etwa Sen 2000; Knecht 2010; Deutscher Bundestag 2013: 299ff.). ${ }^{2}$ Für unsere Studie und allgemeiner für die Verwirklichung zukunftsorientierter Politik ist weiters wichtig, dass ökologische Nachhaltigkeit eine Gestaltungsaufgabe ist, die insofern weit über eine unmittelbar intakte und lebenswerte Umwelt von Individuen oder einer Gesellschaft hinausgeht, als sie auch die ,Externalisierung der negativen Voraussetzungen und Konsequenzen einer naturzerstörerischen Produktions- und Lebensweise berücksichtigen muss (vgl. Brand/Wissen 2017).

Vor diesem Hintergrund geht es uns im Folgenden nicht um eine Diskussion angemessener Indikatoren. Vielmehr fragen wir nach ihrem Stellenwert als Teil der Auseinandersetzungen um ein sozial-ökologisch erweitertes Fortschritts- und Wohlstandsverständnis sowie als Einsatzpunkt einer in diesem Sinn lebensqualitäts- und wohlstandsorientierten Wirtschafts- und Gesellschaftspolitik.

1 Der Fokus auf strategische Möglichkeiten impliziert, dass die zu verankernden Alternativen vor dem Hintergrund bestehender Kräfteverhältnisse und Konflikte relational betrachtet werden. Denn ein sozial-ökologisch erweitertes Fortschritts- und Wohlstandsverständnis und die entsprechenden Politiken implizieren die Notwendigkeit, dominante und aus sozialer wie ökologischer Sicht problematische Verständnisse und Politiken zurückzudrängen.

2 Die Enquete-Kommission, Wachstum, Wohlstand, Lebensqualität ${ }^{\prime}$ des Deutschen Bundestages relativiert die Begriffe ,Wohlstand" und ,Lebensqualität': „Doch schon die Frage, welche Aspekte eindeutig zum Wohlstand gehören, ist schwer zu beantworten. Eine abschließende Liste kann es aufgrund unterschiedlicher Werturteile, Weltanschauungen und Interessen von Individuen zwangsläufig niemals geben. Die Frage, was das ,erfüllte menschliche Leben' ist, beantworten Menschen naturgemäß höchst unterschiedlich [...]. Jeder Versuch, Wohlstand und Lebensqualität ,objektiv' und abschließend zu bestimmen, ist zum Scheitern verurteilt.“ (Deutscher Bundestag 2013: 234)
Dabei werden in einem ersten Schritt die theoretischen und methodischen Grundlagen des Artikels dargestellt (Abschnitt 2), um im Anschluss mit der neueren Indikatorendebatte den zentralen Ausgangspunkt unserer Überlegungen zu skizzieren (Abschnitt 3). Im darauffolgenden Hauptteil rückt mit Blick auf eine breite mediale sowie auf eine politisch-institutionelle Öffentlichkeit die forschungsleitende Frage nach zentralen Barrieren für eine bessere Verankerung lebensqualitäts- und wohlstandsorientierter Politik sowie nach strategischen Möglichkeiten ihrer Überwindung in den Fokus (Abschnitt 4). ${ }^{3}$ Die vielfältigen, in ExpertInneninterviews herausgearbeiteten Politikoptionen des Hauptteils werden alsdann zu einigen theoriegeleiteten Schlussfolgerungen im Hinblick auf die Überwindung der bestehenden Restriktionen für eine sozial und ökologisch nachhaltige Form von Wirtschafts- und Gesellschaftspolitik verdichtet (Abschnitt 5). Abschließende Bemerkungen zur Umkämpftheit von Wohlstand sowie zu offenen Fragen runden den Beitrag ab (Abschnitt 6).

\section{Theoretische und methodische Grundlagen}

Wie oben dargelegt, fokussiert der vorliegende Artikel auf Möglichkeiten und Hürden der Verankerung lebensqualitäts- und wohlstandsorientierter Politik in Österreich. Forschungsleitend ist mithin die Frage, woran diese Verankerung bislang scheitert bzw. wie sie zukünftig gelingen kann. Mit anderen Worten geht es uns also um die Frage, warum Ansätze einer solchen auf einem erweiterten Wohlstandsverständnis basierenden Politik es in Österreich bislang - als Lösungsvorschläge für das Problem einer einseitig am BIP-Wachstum orientierten Wirtschaftspolitik - kaum auf die politische Agenda schaffen, geschweige denn als konkrete Alternativen zum Gegenstand politischer Entscheidungen werden.

3 Von spezifischem Interesse ist neben der breiten medialen bzw. der politisch-institutionellen die wissenschaftliche (Fach-)Öffentlichkeit, die im vorliegenden Artikel jedoch nicht gesondert behandelt werden kann. Als Hürden für eine bessere Verankerung lebensqualitäts- und wohlstandsorientierter Politik lassen sich hier etwa fachliche Herausforderungen (z.B. lückenhafte Datengrundlagen), die fortwirkende Dominanz orthodoxer Paradigmen an den Universitäten sowie fehlende Ressourcen in der außeruniversitären Forschung bestimmen (Griesser/Brand 2016: 44ff.). Für eine vergleichbare Auseinandersetzung mit solchen „Indikatoren-Barrieren“ vgl. etwa Whitby et al. 2014: 29ff.; Bleys/Whitby 2015: 167f. 
In der Politikwissenschaft steht diese Frage im Zentrum von Arbeiten, die im Rahmen der Policybzw. Politikfeldforschung ein besonderes Augenmerk auf Prozesse des Agenda-Settings richten (vgl. Schneider/Janning 2006: 27/50ff.). Auch im Kontext von Untersuchungen zum politischen Impact alternativer Wohlstands- und Fortschrittsindikatoren wurde so in den vergangenen Jahren von einer Reihe von AutorInnen auf einen Klassiker dieser Forschungsrichtung, nämlich auf John Kingdons Multiple-Streams-Ansatz referiert (vgl. Bache 2013; Bache/Reardon 2013, 2016; Griesser/Brand 2016; Whitby et al. 2014). Fokussierend auf die Agenda-Gestaltung als zentraler Phase des Policy-Prozesses geht dieser Ansatz davon aus, dass in Gestalt von Problem-, Policies- und Politics-Streams drei Ströme bzw. Prozesse differenziert werden können, die relativ unabhängig voneinander sind und sich durch jeweils spezifische Entwicklungsmuster und -dynamiken auszeichnen (vgl. Kingdon 1984: 17ff./92ff.).

Auf den Gegenstand des vorliegenden Artikels übertragen bedeutet dies, dass die für die Gestaltung einer politischen Agenda bzw. für die Auswahl entsprechender Alternativen relevanten Faktoren - die Wahrnehmung des Problems (Problem Recognition), das Vorhandensein darauf bezogener Lösungsvorschläge (Policy Proposals), deren Unterstützung durch politische Kräfte (Political Processes) usw. - in relativer Unabhängigkeit voneinander bereits vorhanden sind. Entscheidend ist daher die Verknüpfung dieser Faktoren bzw. der damit verbundenen Prozesse, denn nur wenn dies gelingt - wenn also in Bezug auf eine bestimmte Problemwahrnehmung ein konsistenter Lösungsvorschlag formuliert und dieser von einer machtvollen Interessenkoalition unterstützt wird -, kann das Sachthema auf der politischen Agenda nach oben rücken bzw. dort überhaupt erst erscheinen (vgl. ebd.: 21).

Gemäß der Perspektive Kingdons (1984: 173ff.) hängt dies von zwei zentralen Faktoren ab: Konkret setzt es die Öffnung eines Möglichkeitsfensters (Window of Opportunity) voraus, wodurch sich für sogenannte Policy Entrepreneurs die Gelegenheit ergibt, im Sinne der genannten Verknüpfung etwa für wahrgenommene Problemlagen konkrete Lösungsvorschläge und damit verbundene Interessenkoalitionen ins Spiel zu bringen. Ersteres (d.h. die Öffnung eines Möglichkeitsfensters) verweist im Sinne, objektiver Bedingungen' also auf strukturelle Aspekte, letzteres (d.h. die Aktivitäten von Policy Entrepreneurs) im Sinne ,subjektiver Möglichkeiten' auf handlungsbezogene Aspekte. Mit dem
Konzept der Policy Entrepreneurs werden dabei (individuelle) AkteurInnen aus unterschiedlichen Bereichen (Verwaltung, Wissenschaft usw.) gefasst, die auf die Öffnung eines solchen Möglichkeitsfensters lauern, um mit Blick auf einen potenziellen zukünftigen Nutzen (materieller, solidarischer usw. Art) verschiedene Ressourcen (Geld, Reputation usw.) zu investieren (vgl. ebd.: 188).

Im vorliegenden Artikel wollen wir diesen Gedanken aufgreifen und in zweifacher Form erweitern. Zum ersten verorten wir den Ansatz Kingdons im epistemologischen Rahmen sogenannter interpretativer Policy-Analysen (IPA). Diese unterscheiden sich von älteren Ansätzen im Bereich der Politikfeldforschung vor allem dadurch, dass sie auf den diskursiv vermittelten (d.h. lediglich über sprachliche Zeichen erkenn- und erfassbaren) Charakter sozialer Wirklichkeit insistieren und daraus - konkret aus der grundsätzlichen Bedeutungsoffenheit und semantischen Umkämpftheit sozialer Phänomene - die Relevanz von Deutungskämpfen für politische Prozesse schlussfolgern (vgl. etwa Fischer/Gottweis 2012: 7; Münch 2016: 3ff.). Für den vorliegenden Kontext von Relevanz ist insbesondere die Annahme, dass Policy Entrepreneurs aus dieser Perspektive vor allem mit konkurrierenden Deutungsrahmen bzw. diskursiven Frames ,handeln', die auf politische Sachthemen bezogen und für den Prozess der Agendagestaltung von zentraler Bedeutung sind (vgl. etwa ebd.: 79ff.; Rein/Schön 1993).

Zum anderen erweitern wir den Ansatz Kingdons um eine staats- und institutionentheoretische Perspektive ausgehend von der Annahme, dass das Agenda-Setting keineswegs als offener und von den gesellschaftlichen Verhältnissen losgelöster Prozess zu begreifen ist. Vielmehr unterliegt dieser Prozess, im Zuge dessen Sachthemen auf die politische Agenda gesetzt bzw. in der damit verbundenen Prioritätenliste (nach oben) verschoben werden, vielfältigen, aus besagten Verhältnissen selbst resultierenden Restriktionen (vgl. auch Brand 2013: 430ff.). ${ }^{4}$ Im vorliegenden Zusammenhang wollen wir uns auf zwei Arten von Restriktionen konzentrieren. Diese sind im Hinblick auf die dargelegte Forschungsfrage insofern von besonderer Bedeutung, als sie auf den Prozess der Agenda-

4 Dieser Annahme liegen Überlegungen zugrunde, die andernorts im Sinne einer historisch-materialistischen Policy-Analyse (HMPA) ausformuliert wurden (vgl. Brand 2013; Griesser 2017). 
Abbildung 1: Prozess der Agenda-Gestaltung

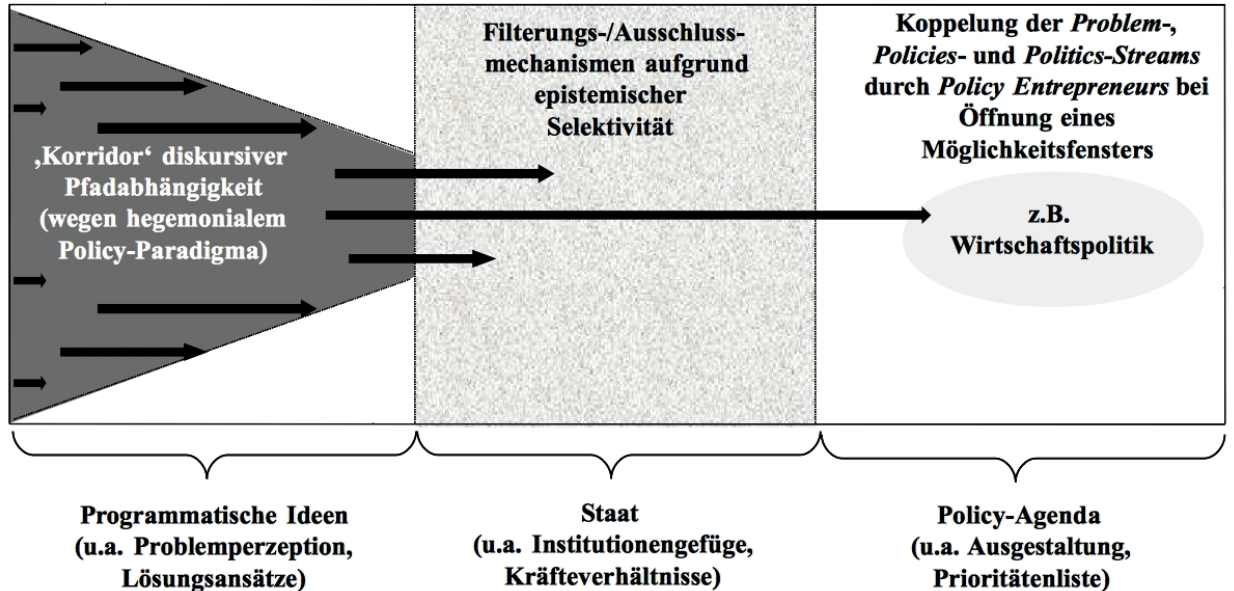

Quelle: Eigene Darstellung

Gestaltung entweder in endogener oder in exogener Form beschränkend einwirken (vgl. Grafik I).

Was die endogen wirksamen Restriktionen anbelangt wurde von neo-institutionalistischer Seite das Konzept der Pfadabhängigkeit (weiter-)entwickelt, um zu erklären, wie aktuelle Entscheidungen das Einschlagen eines bestimmten Entwicklungspfads bedingen und darüber im Sinne pfadabhängiger Entwicklung auch zukünftige Entscheidungen prägen. In historischer Perspektive impliziert dies eine institutionelle Form der Priorisierung von Policies, die sich hinsichtlich des eingeschlagenen Pfads durch Kontinuität gegenüber solchen, die sich durch Diskontinuität auszeichnen. Erklärt wird dieser Mechanismus häufig über Selbstverstärkungseffekte (Increasing Returns), die den relativen Nutzen bestimmter bzw. die relativen Kosten alternativer Entscheidungen mit der Zeit in selbstverstärkender Weise erhöhen und darüber Wandel unwahrscheinlich machen (vgl. etwa Pierson 2000; Schneider/Janning 2006: 34f.).

Im Gegensatz dazu schlagen wir aus der dargelegten Perspektive einer interpretativen Policy-Analyse vor, diesen Bias zugunsten bestimmter Problemwahrnehmungen bzw. Lösungsvorschläge unter Rekurs auf diskursive Faktoren zu erklären. Das heißt, wir führen die im Prozess der Agenda-Gestaltung evidente Priorisierung bestimmter programmatischer Ideen (vgl. Schmidt 2011: 108ff.) auf eine Form der diskursiven Pfadabhängigkeit zurück. Wir nehmen also an, dass manche Ideen (z.B. Wachstum = Wohlstand) gegenüber anderen Ideen (z.B. geringe Vermögensungleichheit bzw. hohe Biodiversität = Wohlstand) deshalb priorisiert werden, weil sie mit institutionell sedimen- tierten Wissensbeständen in Kontinuität stehen und als entsprechend passfähig gelten. Verantwortlich für die variierende Passfähigkeit programmatischer Ideen sind aus der hier gewählten Perspektive das jeweils hegemoniale Policy-Paradigma (z.B. wachstums- versus wohlstandsorientiertes Paradigma $)^{5}$ sowie die dadurch abgesteckten ,Korridore von als (im epistemologischen Sinn) angemessen und vernünftig geltender Politik (vgl. etwa Brand 2013: 433f.).

Was schließlich die Frage der exogenen, also von außen auf den Prozess der Agenda-Gestaltung einwirkenden Restriktionen betrifft, geht es um jene Selektivität politischer Institutionen, die - wie Claus Offe (1972/2006: 105) es formulierte - „die nicht-zufällige (d.h. systematische) Restriktion eines Möglichkeitsraumes“ bedingt. Dem zugrunde liegt die Überlegung, dass nicht für alle Sachthemen der Weg auf die politische Agenda bzw. an die Spitze politischer Prioritätenlisten gleichermaßen offen steht, sondern dass deren Chancen aufgrund einer Reihe institutionalisierter Filterungs- und Ausschlussregeln variieren. Im Extremfall wird - im Sinne der Nicht-Entscheidungen (Non-Decisions) von Peter Bachrach und Morton S. Baratz (1975)

5 Peter A. Hall (1993) hat das von Thomas S. Kuhn im Bereich der Wissenschaftstheorie geprägte Konzept des Paradigmas für die Politikfeldforschung produktiv gemacht. Unter einem Policy Paradigm versteht Hall dabei ein „framework of ideas [...] that specifies not only the goals of policy and the kind of instruments that can be used to attain them, but also the very nature of the problems they are meant to be addressing" (ebd.: 279; vgl. dazu auch Bache/Reardon 2016: 9f./26ff.; zum wachstumsorientierten Paradigma Schmelzer 2016). 
- so verhindert, dass manche Themen überhaupt zum Gegenstand politischer Entscheidungen werden (vgl. auch Schneider/Janning 2006: 55f.).

Konkret hängen besagte Chancen von den Möglichkeiten des Zugangs zu bzw. der Kontrolle über Ressourcen ab, die für verschiedene politische Kräfte in jeweils spezifischer, nämlich "strategisch-selektiver" (Jessop 2002: 40) Form ausgestaltet sind. Wir gehen also davon aus, dass in die politischen Institutionen selbst Mechanismen eingeschrieben sind, die eine Filterungs- bzw. Ausschlussfunktion gegenüber den von bestimmten Akteuren verfolgten Interessen, Strategien, Zeithorizonten usw. erfüllen. Aus der Perspektive einer interpretativen Policy-Analyse interessieren uns in diesem Zusammenhang in erster Linie "diskursive“ (ebd.: 93) bzw. „epistemische Selektivitäten“ (Brand/ Vadrot 2013: 207ff.) im Sinne solcher Mechanismen, die spezifische Wissensformen, Problemperzeptionen und Lösungsvorschläge gegenüber anderen präferieren (vgl. ebd.; Brand 2013: 432f.).

In methodischer Hinsicht basiert der Artikel auf 20 rund einstündigen ExpertInneninterviews, die zwischen März und September 2016 in Wien durchgeführt wurden. Wie bei leitfadengestützten Interviews üblich (vgl. etwa Bogner et al. 2014; Gläser/Laudel 2010: 4off.) orientierten sich diese an einem vorab entwickelten Fragenkatalog, der als eine Art Richtschnur diente und die Vergleichbarkeit der erhobenen Daten garantieren sollte. Deren Auswertung erfolgte mithilfe von Instrumenten aus dem Bereich der qualitativen Inhaltsanalyse (vgl. etwa ebd.: 43fff.; Bogner et al. 2014: 17ff./71ff.). Konkret wurden die vollständig transkribierten und anonymisierten Interviews im Sinne textförmiger Daten in einem ersten Schritt mithilfe eines Analyserasters entlang ex ante entwickelter Kategorien einer Feinanalyse unterzogen. In einem nachfolgenden Schritt ging es darum, die so gewonnenen Informationen in aggregierter und vom Ursprungstext abstrahierter Form aufzubereiten und im Rahmen einer Gesamtanalyse interpretativ-analytisch auszuwerten.

Bei der Auswahl der ExpertInnen wurde eine ausgewogene Repräsentation von fünf StakeholderGruppen angestrebt, die im Hinblick auf unser Erkenntnisinteresse als zentrale (Kollektiv-)Akteure betrachtet werden können. Konkret interviewt wurden dabei: (a) sieben ExpertInnen aus dem Bereich Politik und Verwaltung (aus den Ressorts Umwelt, Soziales und Wirtschaft sowie aus dem Bundeskanzleramt/ BKA); (b) drei ExpertInnen aus dem Bereich der (angewandten) Wissenschaft inklusive Bundesanstalt für Statistik (Statistik Austria, Wirtschaftsforschungsinstitute); (c) fünf ExpertInnen aus dem Bereich Verbände und Gewerkschaften inklusive verbandsnaher Einrichtungen (Kammer für Arbeiter und Angestellte/ AK, Österreichischer Gewerkschaftsbund/ÖGB, Wirtschaftskammer Österreich/WKO, Industriellenvereinigung/IV); (d) drei ExpertInnen von NGOs aus den Bereichen Wirtschaft, Umwelt und Soziales (Attac, Armutskonferenz usw.); und (e) zwei ExpertInnen aus dem Bereich Medien und Journalismus (aus den Ressorts Wirtschaft und Gesellschaft). ${ }^{6}$

\section{Zentrale Etappen der neueren Indikatoren- debatte im Überblick}

In der Nachkriegszeit avancierte die in den 1930er-Jahren entwickelte Kennziffer des Bruttoinlandsprodukts (BIP) bzw. deren Varianten (z.B. Bruttonationaleinkommen, Bruttosozialprodukt) - aufbauend auf Vorarbeiten, wie sie seit dem 17. Jahrhundert von Ökonomen wie William Petty und später vor allem Colin Clark und Simon Kuznets unternommen wurden (vgl. etwa Lepenies 2013; Fioramonti 2013) - zum weltweit bedeutendsten Maßstab gesellschaftlichen Wohlstands und Fortschritts sowie damit assoziierter ,Entwicklung. Beinahe ebenso lang wie die Geschichte des Aufstiegs des BIP währt jedoch jene seiner Problematisierung und Kritik (vgl. etwa ebd.: 5off.; Chaloupek/Feigl 2012). Einen ersten Höhepunkt erreichte die daran anschließende Diskussion in den 1970er- und 1980er-Jahren. Wie Scheiblecker et al. (2011: 715) konstatieren, gingen die wesentlichen Impulse damals jedoch von der Wirtschaftswissenschaft aus, wohingegen das Wiederaufflammen der

6 Das Ungleichgewicht zugunsten von WirtschaftswissenschafterInnen im Bereich der Wissenschaft (nahezu alle interviewten WissenschafterInnen waren ÖkonomInnen) sowie zugunsten von mit wirtschaftspolitischen Fragen beschäftigten Personen im Bereich der Politik und Verwaltung (nahezu alle interviewten PolitikerInnen bzw. BeamtInnen waren - wenn auch in unterschiedlichen Ressorts - mit Wirtschaftsagenden befasst) ist dem Design des Forschungsprojekts geschuldet, das dem vorliegenden Artikel zugrunde liegt. Inwiefern diese Auswahl der InterviewpartnerInnen lediglich bestehende Ungleichgewichte im Bereich wirtschaftspolitischer Entscheidungsprozesse reflektiert oder diese zuspitzt - und darüber auch die Forschungsergebnisse verzerrt -, kann hier nicht abschließend beantwortet werden. Eine stärkere Berücksichtigung von ExpertInnen aus anderen Disziplinen und Feldern im Rahmen zukünftiger Forschungsprojekte wäre aber in jedem Fall wünschenswert. 
Tabelle 1: Die neuere Indikatorendebatte im Überblick

\begin{tabular}{|c|c|c|}
\hline \multicolumn{3}{|c|}{ Stiglitz-Sen-Fitoussi-Report (Commission on the Measurement of Economic Performance and ...) } \\
\hline $\begin{array}{l}\text { Materieller Wohlstand } \\
\text { (Classical GDP issues) }\end{array}$ & $\begin{array}{l}\text { Lebensqualität } \\
\text { (Quality of life) }\end{array}$ & $\begin{array}{l}\text { Nachhaltigkeit } \\
\text { (Sustainability) }\end{array}$ \\
\hline $\begin{array}{c}\text { z.B. Akzentuierung der } \\
\text { Haushaltsperspektive; Entwicklung } \\
\text { von Kennzahlen für die } \\
\text { Einkommens-, Konsum- und } \\
\text { Vermögensverteilung }\end{array}$ & $\begin{array}{l}\text { z.B. Erhebung von Daten zum } \\
\text { subjektiven Wohlergehen; Erfassung } \\
\text { von Ungleichheiten, u.a. zwischen } \\
\text { sozioökonomischen Gruppen und } \\
\text { Geschlechtern }\end{array}$ & $\begin{array}{l}\text { z.B. Entwicklung eines } \\
\text { Indikatorensystems für die Bewertung } \\
\text { von Nachhaltigkeit; Bestimmung von } \\
\text { Kennziffern für Veränderungen im } \\
\text { Bestand, u.a. natürlicher Ressourcen }\end{array}$ \\
\hline \multicolumn{3}{|c|}{ Bericht der Sponsorship Group des obersten Statistikgremiums der EU (ESSC) } \\
\hline $\begin{array}{c}\text { Materieller Wohlstand } \\
\text { (The Household Perspective) }\end{array}$ & $\begin{array}{l}\text { Lebensqualität (Multidimensional } \\
\text { Measurement of Quality of Life) }\end{array}$ & $\begin{array}{c}\text { Umweltorientierte Nachhaltigkeit } \\
\text { (Environmental Sustainability) }\end{array}$ \\
\hline $\begin{array}{l}\text { z.B. Erhebung von Daten zur } \\
\text { Verteilung von Einkommen, Konsum } \\
\text { und Vermögen; Ausweitung der } \\
\text { Messung von Einkommen auf nicht- } \\
\text { marktvermittelte Tätigkeiten }\end{array}$ & $\begin{array}{l}\text { z.B. Nutzung von EU-Statistiken zu } \\
\text { Einkommen und Lebensbedingungen } \\
\text { als Kerninstrument; Abdeckung } \\
\text { weiterer Dimensionen durch Rück- } \\
\text { griff auf zusätzliche Datenquellen }\end{array}$ & $\begin{array}{l}\text { z.B. Weiterentwicklung von mit dem } \\
\text { Klimawandel verbundenen } \\
\text { Indikatoren; Entwicklung von Konten } \\
\text { für Energieflüsse und von darauf } \\
\text { basierenden Indikatoren }\end{array}$ \\
\hline \multicolumn{3}{|c|}{ Expertise im Auftrag des Deutsch-Französischen Ministerrats (SVR/CAE) } \\
\hline $\begin{array}{l}\text { Materieller Wohlstand und } \\
\text { Wirtschaftsleistung }\end{array}$ & Lebensqualität & $\begin{array}{c}\text { Ökologische und wirtschaftliche } \\
\text { Nachhaltigkeit }\end{array}$ \\
\hline $\begin{array}{l}\text { Sechs Indikatoren, z.B. BIP pro Kopf; } \\
\text { Beschäftigungsquote der 15- bis 64- } \\
\text { Jährigen; Quotient aus dem obersten } \\
\text { und untersten Quintil der } \\
\text { Einkommensanteile }\end{array}$ & $\begin{array}{l}\text { Sieben Dimensionen mit je einem } \\
\text { Leitindikator, z.B. } \\
\text { Umweltbedingungen: Belastung der } \\
\text { städtischen Bevölkerung durch } \\
\text { Luftverschmutzung mit Feinstaub }\end{array}$ & $\begin{array}{l}\text { Zwölf Indikatoren, z.B. Niveau der } \\
\text { Treibhausgasemissionen; inländischer } \\
\text { Materialverbrauch pro Kopf; } \\
\text { konjunkturbereinigter } \\
\text { Finanzierungssaldo relativ zum BIP }\end{array}$ \\
\hline \multicolumn{3}{|c|}{ OECD-Projekt How's Life? Measuring Well-Being im Rahmen der Better-Life-Initiative } \\
\hline $\begin{array}{l}\text { Materielle Lebensbedingungen } \\
\text { (Material Living Conditions) }\end{array}$ & $\begin{array}{l}\text { Lebensqualität } \\
\text { (Quality of Life) }\end{array}$ & $\begin{array}{l}\text { Nachhaltigkeit } \\
\text { (Sustainability) }\end{array}$ \\
\hline $\begin{array}{l}\text { Sieben Indikatoren, z.B. verfügbares } \\
\text { Haushaltsnettoeinkommen; } \\
\text { Wohnkosten in Relation zum } \\
\text { Haushaltsbruttoeinkommen; } \\
\text { Langzeitarbeitslosenquote }\end{array}$ & $\begin{array}{l}15 \text { Indikatoren, z.B. } \\
\text { Lebenserwartung; Zeit für Freizeit- } \\
\text { aktivitäten und persönliches Wohl- } \\
\text { befinden; Wahlbeteiligung; Konzen- } \\
\text { tration von Feinstaub in Städten }\end{array}$ & $\begin{array}{l}\text { Die Nachhaltigkeit von natürlichem, } \\
\text { ökonomischem, Human- und } \\
\text { Sozialkapital (keine separaten } \\
\text { Kennziffern) }\end{array}$ \\
\hline \multicolumn{3}{|c|}{ Statistik-Austria-Projekt Wie geht's Österreich? } \\
\hline Materieller Wohlstand & Lebensqualität & Umweltorientierte Nachhaltigkeit \\
\hline $\begin{array}{l}\text { Acht Indikatoren, z.B. BIP pro Kopf; } \\
\text { Arbeitsproduktivität; Entwicklung } \\
\text { hoher und niedriger } \\
\text { Bruttojahreseinkommen; Zeitaufwand } \\
\text { für unbezahlte Arbeit }\end{array}$ & $\begin{array}{l}\text { Elf Indikatoren, z.B. Armuts- und } \\
\text { Ausgrenzungsgefährdung; } \\
\text { Tragfähigkeit sozialer Beziehungen; } \\
\text { Vertrauen in das politische System; } \\
\text { subjektive Umweltbelastung }\end{array}$ & $\begin{array}{l}\text { Zwölf Indikatoren, z.B. inländischer } \\
\text { Materialverbrauch; } \\
\text { Treibhausgasemissionen; Anteil der } \\
\text { erneuerbaren Energieträger; } \\
\text { Ökosteueranteile }\end{array}$ \\
\hline
\end{tabular}

Quelle: Eigene Darstellung

Debatte in den 200oer- und 2010er-Jahren in erster Linie von der Wirtschaftspolitik vorangetrieben wurde.

Dies soll im Folgenden konkretisiert werden, indem wir - ohne Anspruch auf Vollständigkeit einige zentrale Etappen der Debatte seit Ende der 2oooer-Jahre in Erinnerung rufen (siehe Tabelle I). Konkret werden auf internationaler Ebene entwickelte Projekte im Anschluss an die Commission on the Measurement of Economic Performance and Social Progress sowie deren Rezeption in Österreich dargelegt. ${ }^{7}$ Darüber soll die Grundlage für den empirischen Teil des Artikels entwickelt werden, in dem die Frage nach

7 Das impliziert, dass eine Vielzahl von Projekten im Bereich der Indikatorendebatte - insbesondere historisch ältere (z.B. der Human Development Index der Vereinten Nationen) und geografisch entlegenere (z.B. der Gross National Happiness Indicator in Bhutan) - hier unberücksichtigt bleiben muss (für einen knappen Überblick vgl. etwa Diefenbacher/Zieschank 2011; Deutscher Bundestag 2013: 299-336). 
den strategischen Möglichkeiten einer besseren Verankerung lebensqualitäts- und wohlstandsorientierter Politik im Zentrum steht.

Für den Verlauf der neueren Indikatorendebatte von zentraler Bedeutung war - wie angedeutet - die Einsetzung der Commission on the Measurement of Economic Performance and Social Progress durch den damaligen französischen Staatspräsidenten Nicolas Sarkozy im Jahr 2008. Der 2009 präsentierte Endbericht der Kommission unter der Leitung von Joseph E. Stiglitz, Amartya Sen und Jean-Paul Fitoussi intensivierte nämlich nicht bloß die Debatte um eine Erneuerung des dominanten Wohlstands- und Fortschrittsverständnisses, sondern gab auch Impulse für ihren weiteren Verlauf (vgl. etwa Diefenbacher/Zieschank 2011: 51ff.). Das wird allein schon am Umstand ersichtlich, dass praktisch alle in den folgenden Jahren initiierten Projekte in diesem Bereich auf den Stiglitz-Sen-Fitoussi-Report (SSF-Report) Bezug nahmen (vgl. etwa Europäische Kommission 2009: 3f.; SVR/CAE 2010: 4/10f.; OECD 2011: 3/18; Deutscher Bundestag 2013: 318ff.; Statistik Austria 2015: 14/34).

Der SSF-Report formuliert dabei keinen eigenen Vorschlag für ein Indikatorenset o.Ä., sondern arbeitet in Bezug auf drei Schwerpunktthemen zentrale Problematiken heraus und entwickelt in Gestalt konzeptioneller Empfehlungen darauf bezogene Lösungsvorschläge. Die drei genannten Themenbereiche sind (a) materieller Wohlstand (Classical GDP Issues), für den z.B. eine stärkere Akzentuierung der Haushaltsperspektive sowie die Entwicklung von Kennzahlen, u.a. für die Einkommens- und Vermögensverteilung, empfohlen werden; (b) Lebensqualität (Quality of Life), für den z.B. die Erhebung von Daten zum subjektiven Wohlbefinden sowie die Erfassung von Ungleichheiten, u.a. zwischen sozioökonomischen Gruppen und Geschlechtern, gefordert werden; sowie (c) Nachhaltigkeit (Sustainability), für den z.B. die Entwicklung eines eigenen Indikatorensystems für die Bewertung von Nachhaltigkeit sowie die Bestimmung von Kennziffern für Veränderungen im Bestand, u.a. natürlicher Ressourcen, angeregt werden (vgl. Stiglitz et al. 2009).

Wie oben bereits erwähnt und von der Kommission intendiert - „[t]he Commission regards its report as opening a discussion rather than closing it" (ebd.: 18) -, wurden die von ihrem Abschlussbericht ausgehenden Impulse in den folgenden Jahren von zahlreichen Organisationen und Institutionen aufgenommen. Unmittelbar gilt dies für eine im Auftrag des
Deutsch-Französischen Ministerrats erstellte Expertise des deutschen Sachverständigenrats zur Begutachtung der gesamtwirtschaftlichen Entwicklung (SVR) sowie seines französischen Pendants, des Conseil d'Analyse Économique (CAE), der ausgehend von den Empfehlungen des SSF-Reports ein - wie es im Untertitel heißt - „umfassendes Indikatorensystem“ entwickelt (vgl. etwa Bache/Reardon 2013: 904). Die insgesamt 25 Kennziffern finden sich dabei den drei oben dargestellten Säulen zugeordnet, d.h. (a) dem materiellen Wohlstand und der Wirtschaftsleistung (z.B. BIP pro Kopf; Beschäftigungsquote der 15- bis 64-Jährigen); (b) der Lebensqualität (z.B. Mitspracherecht und Verantwortlichkeit; Belastung der städtischen Bevölkerung durch Luftverschmutzung mit Feinstaub); sowie (c) der ökologischen und wirtschaftlichen Nachhaltigkeit (z.B. Konjunkturbereinigter Finanzierungssaldo in Relation zum BIP; Niveau der Treibhausgasemissionen) (vgl. SVR/CAE 2010: 12ff.).

Auf internationaler Ebene war es vor allem die OECD, die bereits die Arbeit am SSF-Report aktiv begleitet hat und späterhin die davon ausgehenden Impulse aufgriff (vgl. etwa Pesendorfer/Leitner 2010: 539f.). Parallel dazu arbeitete die Organisation schon seit Jahren eine eigene Agenda zum Thema aus, was 2011 in die aus Anlass ihres 50-jährigen Bestehens initiierte Better Life-Initiative mündete. Diese umfasste u.a. das Projekte How's Life?, in dessen Rahmen seit 2011 im Zwei-Jahres-Rhythmus ein Bericht in Anwendung des Better Life-Indikatorensets der OECD erscheint. Besagtes Set umfasst elf Teilbereiche mit jeweils einem bis vier Indikatoren. Diese werden wiederum zwei Dimensionen zugeordnet, nämlich (a) den materiellen Lebensbedingungen (z.B. bereinigtes verfügbares Haushaltsnettoeinkommen; Langzeitarbeitslosenquote) und (b) der Lebensqualität (z.B. Lebenserwartung; Wahlbeteiligung), wobei auch hier mit (c) der Nachhaltigkeit von natürlichem, ökonomischem, Human- und Sozialkapital eine dritte, für die Wohlfahrt zukünftiger Generationen relevante Dimension bestimmt wird (vgl. etwa OECD 2011: 21ff.).

Auf EU-Ebene veröffentlichte die Kommission referierend auf eine bereits 2007 unter dem Titel Beyond GDP ausgetragene Konferenz - im August 2009 (nunmehr unter dem neuen Titel GDP and Beyond: Measuring Progress in a Changing World) eine Roadmap for Action (vgl. Europäische Kommission 2009). In den darauffolgenden Jahren starteten diverse Institutionen, Gremien und Behörden Initiativen zur Umsetzung des hier entwickelten Fahrplans für eine Erweiterung des 
BIP (vgl. etwa Bache 2013: 27ff.). ${ }^{8}$ Dazu zählte zuvorderst die vom European Statistical System Committee (ESSC), dem obersten Statistikgremium der EU, im Frühjahr 2010 eingesetzte Sponsorship Group on Measuring Progress, Well-being and Sustainable Development. Deren Auftrag bestand darin, die mit GDP and Beyond sowie mit dem SSF-Report verbundenen Vorgaben in konkrete Arbeitsprogramme für das ESSC zu überführen (vgl. etwa Pesendorfer/Leitner 2010: 539f.).

Der als Sponsorship Report bekannt gewordene Abschlussbericht umfasst entsprechend Empfehlungen für die drei Bereiche (a) des materiellen Wohlstands (z.B. Erhebung von Daten zur Verteilung von Einkommen, Konsum und Vermögen; Ausweitung der Messung von Einkommen auf nicht-marktvermittelte Tätigkeiten in Privathaushalten und in der Freizeit); (b) der Lebensqualität (z.B. Nutzung von EU-Statistiken zu Einkommen und Lebensbedingungen als Kerninstrument; Abdeckung weiterer Dimensionen durch Rückgriff auf zusätzliche Datenquellen); sowie (c) der umweltorientierten Nachhaltigkeit (z.B. Weiterentwicklung von mit dem Klimawandel verbundenen Indikatoren; Entwicklung von Konten für Energieflüsse und von darauf basierenden Indikatoren) (vgl. ESSC 2011: 15ff.).

In Österreich schließlich wurde vor allem seitens der nationalen Statistikbehörde Statistik Austria als ausgegliederte Bundesanstalt öffentlichen Rechts auf die Debatte reagiert. ${ }^{9}$ Konkret begann dort im Jahr 2011 eine abteilungsübergreifende ExpertInnengruppe basierend auf den Empfehlungen des SSF-Reports sowie des Abschlussberichts der Sponsorship Group mit der Ausarbeitung eines eigenen Indikatorensets. Dieses wurde unter dem Namen Wie geht's Österreich? (WGÖ?) erstmals im Rahmen des Statistiktags 2012 öffentlich präsentiert. Seitdem publiziert die Statistik Austria einmal jährlich ihren WGÖ?-Bericht, der die Entwicklungen von - neben dem BIP - 30 Schlüssel-

8 In den Augen der Europäischen Kommission (2013: 32) markieren vor allem die Europa-2020-Strategie für Beschäftigung und Wachstum sowie das damit verbundene Steuerungsinstrument des Europäischen Semesters „early examples of the practical implementation of the GDP and Beyond initiative", werden damit doch neben wirtschaftlichen auch soziale und ökologische Ziele in indikatorenbasierter Form verfolgt.

9 Als ein weiterer wichtiger Akteur im Kontext der neueren Indikatorendebatte kann das Österreichische Institut für Wirtschaftsforschung (WIFO) bestimmt werden (vgl. etwa Griesser/Brand 2016: 28ff./33f.). indikatoren und einer Reihe weiterer Subindikatoren dokumentiert und durch ein externes ExpertInnengremium jeweils in kurz- und langfristiger Perspektive bewerten lässt (vgl. etwa Pesendorfer et al. 2012).

Auch die WGÖ?-Indikatoren werden dabei drei Dimensionen zugeordnet, nämlich (a) dem materiellen Wohlstand (z.B. BIP pro Kopf; Entwicklung hoher und niedriger Bruttojahreseinkommen; Zeitaufwand für unbezahlte Arbeit); (b) der Lebensqualität (z.B. Armuts- und Ausgrenzungsgefährdung; Tragfähigkeit sozialer Beziehungen; Vertrauen in das politische System); und (c) der umweltorientierten Nachhaltigkeit (z.B. Treibhausgasemissionen; Anteil der erneuerbaren Energieträger; Umweltschutzausgaben) (vgl. Statistik Austria 2015: 15ff.).

\section{Probleme und Möglichkeiten sozial und ökologisch nachhaltiger Politik in Österreich}

Auf der Basis der qualitativ-inhaltsanalytischen Auswertung der ExpertInneninterviews sollen im Folgenden jene Faktoren dargestellt werden, die aus deren Perspektive wesentlich für die (mangelhafte) institutionelle und öffentliche Aufmerksamkeit für alternative Kennziffern, für ein damit verbundenes Wohlstands- und Fortschrittsverständnis bzw. für eine darauf basierende Wirtschafts- und Gesellschaftspolitik in Österreich verantwortlich sind..$^{10}$ Dabei wurde versucht, das aus den Interviews extrahierte Material zu jeweils drei zentralen Problem- bzw. darauf bezogenen Lösungskomplexen $\mathrm{zu}$ verdichten und diese thesenförmig zuzuspitzen (vgl. Tabelle II).

Sortiert werden die Ergebnisse entlang der Achse von zwei Öffentlichkeiten, nämlich einer breiten (medialen) und einer politisch-institutionellen Öffentlichkeit, die grob den beiden Teilbereichen - d.h. der Zivilgesellschaft im weiteren und der politischen Gesellschaft im engeren Sinn - des integralen Staates im Sinne Antonio Gramscis (1991ff.: 783) entsprechen. Im

10 Die Relation zwischen den drei genannten Schritten - Aufmerksamkeit für alternative Indikatoren, Erweiterung des Wohlstands- und Fortschrittsverständnisses, Verankerung von lebensqualitäts- und wohlstandsorientierter Politik - wird hier nicht im Sinne eines Automatismus, sondern im Sinne einer Potenzialität begriffen. Alternative Indikatoren fungierten potenziell also als Träger eines erweiterten Verständnisses gesellschaftlichen Wohlstands und Fortschritts und können so auch zum Einsatzpunkt einer entsprechenden Politik werden (ohne dass Letzteres aus Ersterem automatisch folgen würde). 
Tabelle 2: Deutungsmuster der Verankerung sozial und ökologisch nachhaltiger Politik

\begin{tabular}{|c|c|c|}
\hline & Problemformulierung & Lösungsansatz \\
\hline \multicolumn{3}{|c|}{ (I) Breite (mediale) Öffentlichkeit } \\
\hline Themenbereich 1 & $\begin{array}{l}\text { Mangelhaftes Aufgreifen und Vermitteln } \\
\text { des (u.a. von } W G O ̈ ? \text { ausgehenden) } \\
\text { Impulses durch verschiedene politische } \\
\text { (Kollektiv-)Akteure }\end{array}$ & $\begin{array}{l}\text { Vielfältige Formen der diskursiven } \\
\text { Vermittlung durch verschiedene Akteure } \\
\text { mittels enger Lobbying- oder breiter } \\
\text { Beteiligungsverfahren }\end{array}$ \\
\hline Themenbereich 2 & $\begin{array}{l}\text { Problem der Vermittlung komplexer } \\
\text { Indikatorensets wie } W G O ̈ ? \text { (in Relation } \\
\text { zum BIP) über Massenmedien und } \\
\text { andere Kanäle }\end{array}$ & $\begin{array}{l}\text { Bemühen um die Balance zwischen } \\
\text { Komplexität und Überschaubarkeit bzw. } \\
\text { die Aufbereitung, Legitimation und } \\
\text { narrative Vermittlung der Daten }\end{array}$ \\
\hline Themenbereich 3 & $\begin{array}{l}\text { Mit der medialen Situation (in Ö) } \\
\text { verbundene Probleme (u.a. WGÖ?- } \\
\text { Erscheinungsrhythmen) sowie der } \\
\text { Umstand, dass Wandel Zeit braucht }\end{array}$ & $\begin{array}{l}\text { Einzelmaßnahmen in Bezug auf Medien } \\
\text { (u.a. kürzere Intervalle des Erscheinens) } \\
\text { sowie systematische und ausdauernde } \\
\text { Thematisierung }\end{array}$ \\
\hline \multicolumn{3}{|c|}{ (II) Politisch-institutionelle Öffentlichkeit } \\
\hline Themenbereich 1 & $\begin{array}{l}\text { Divergierende Interessenlagen und } \\
\text { Machtasymmetrien in Bezug auf } \\
\text { alternative Indikatoren und auf } \\
\text { wohlstandsorientierte Politik }\end{array}$ & $\begin{array}{l}\text { Ausgehend von Indikatoren politische } \\
\text { Kontroversen über gesellschaftliche Ziele } \\
\text { und damit verbundene } \\
\text { Konflikte/Synergien austragen }\end{array}$ \\
\hline Themenbereich 2 & $\begin{array}{c}\text { Institutionelle Verankerung dominanter } \\
\text { Vorstellungen von Wohlstand/Fortschritt, } \\
\text { die bestimmte Indikatoren auf- bzw. } \\
\text { abwertet }\end{array}$ & $\begin{array}{l}\text { Institutionelle Verankerung neuer } \\
\text { gesellschaftlicher Prioritäten/Ziele und } \\
\text { (Selbst-)Verpflichtung der Politik auf } \\
\text { deren Erreichung }\end{array}$ \\
\hline Themenbereich 3 & $\begin{array}{c}\text { Dominantes Ressortdenken verhindert } \\
\text { Entwicklung integraler Ansätze (u.a. } \\
\text { auch wegen Fehlen von Politikberatungs- } \\
\text { Gremien) }\end{array}$ & $\begin{array}{l}\text { Ressortübergreifende Koordination (z.B. } \\
\text { durch BKA) zwecks Erarbeitung eines } \\
\text { Gesamtkonzepts (unterstützt durch } \\
\text { Gremium wie Wohlstandsrat) }\end{array}$ \\
\hline
\end{tabular}

Quelle: Eigene Darstellung

anschließenden Kapitel werden alsdann unter Rekurs auf den einleitend dargelegten Theorierahmen aus den hier präsentierten Ergebnissen der empirischen Forschung zentrale Schlussfolgerungen abgeleitet.

\subsection{Breite (mediale) Öffentlichkeit}

Problemformulierung I.1: Die Verankerung in einer breiten (medialen) Öffentlichkeit scheitere zum ersten daran, dass der von Initiativen wie Wie geht's Österreich? (WGÖ?) ausgehende Impuls in unzureichender Form von unterschiedlichen (Kollektiv-)Akteuren aufgegriffen und diskursiv in die jeweiligen Bereiche der Gesellschaft hinein vermittelt werde. Denn obschon solche Initiativen eine Sichtbar- und Bewusstmachung von Entwicklungen in verschiedenen Dimensionen gesellschaftlichen Fortschritts und Wohlstands befördern (vgl. Interview Politik 05), scheitern sie doch daran - wie ein Verbandsvertreter es formuliert -, „den Diskurs auch zu organisieren“ (Interview Verbände 03). Dafür verantwortlich zeichnet aus der Perspektive mancher InterviewpartnerInnen auch die mangelhafte
Einbeziehung von ,neuen' Akteuren wie bspw. NGOs in die Entwicklung und Verbreitung der Initiative (vgl. etwa Interviews NGOs 02, NGOs 03).

Lösungsansatz I.1: Eine bessere Verankerung in einer breiten (medialen) Öffentlichkeit sei in diesem Zusammenhang zu erreichen, wenn Akteure auf politischer Ebene, aber auch auf Ebene von Verbänden, NGOs usw. die mit Initiativen wie WGÖ? verbundenen Impulse aufgreifen und in vielfältiger Form in unterschiedliche Teilöffentlichkeiten hinein vermitteln würden (vgl. etwa Interviews Verbände o1, Verbände o3). Was die Methode anbelangt, mit deren Hilfe die alternativen Indikatoren bzw. das darauf basierende Fortschritts- und Wohlstandsverständnis zu verbreiten bzw. weiterzuentwickeln wären, setzen manche auf enge Lobbyingstrategien mit einem relativ kleinen Kreis ,traditioneller' Adressaten (Politik, Verwaltung, Verbände, Wissenschaft) (vgl. etwa Interview Politik o7). Andere hingegen plädieren für breite Beteiligungsverfahren unter Einbeziehung einer Vielzahl von vor allem ,neuen' Akteuren (vgl. etwa Interviews Medien 01, NGOs 02, NGOs 03), nicht zuletzt da die „großen 
gesellschaftlichen Umbrüche immer neue Akteure verlangen" (Interview Wissenschaft 02). ${ }^{1 "}$

Problemformulierung I.2: Eine zweite Hürde für die Verankerung in einer breiten (medialen) Öffentlichkeit bestehe darin, dass ein - in Relation zum BIP in seiner Einfachheit und Schlichtheit - komplexes Indikatorenset wie WGÖ? bzw. ein entsprechend erweitertes Wohlstands- und Fortschrittsverständnis schwer zu vermitteln sei (vgl. etwa Interviews Politik 04, Politik 05). Manche gehen in diesem Kontext gar von einem grundsätzlichen „Trade-off“ (Interview Verbände 05) zwischen Komplexität und Vermittelbar- bzw. Verständlichkeit aus. Dies gelte vor allem für die Massenmedien mit ihrem spezifischen Anforderungsprofil: „Es ist schwer, News daraus zu machen: Also uns geht es besser bei zwölf von 17 Indikatoren, das ist schon eine ganz schwere Botschaft [...]. Wenn es um eine Schlagzeile geht, gewinnt das BIP weiterhin." (Interview Wissenschaft 02) Aber auch über andere Kanäle gelinge die Vermittlung der Debatte jenseits einschlägiger Fachkreise bislang zu wenig (vgl. etwa Interviews NGOs 02, Verbände 02).

Lösungsansatz I.2: Was die Überwindung dieser zweiten Hürde betrifft, gehe es - angesichts der Notwendigkeit eines (ggf. zu verkleinernden) Sets an Indikatoren - darum, sich fortwährend um eine Balance zwischen Komplexität und Überschaubarkeit zu bemühen (vgl. etwa Interviews Wissenschaft o1, Wissenschaft 03). Zudem sei die Aufbereitung und Analyse der Daten, aber auch - wie unter Verweis auf den SSFReport betont wird - deren (fachliche) Legitimation von zentraler Bedeutung (vgl. etwa Interviews Politik 01, Verbände 03, Politik 04, Politik 05). Am bedeutsamsten erscheint vielen jedoch die narrative Vermittlung des Themas in Gestalt einer neuen ,Erzählung bzw. ,Theorie‘. Eine Expertin aus dem Bereich Politik und Verwaltung formuliert es folgendermaßen:

11 Eine weitere Differenz ergibt sich in diesem Zusammenhang aus dem Umstand, dass manche den Zweck einer solchen Beteiligungsstrategie primär in der Dissemination des bereits bestehenden Indikatorensets sehen bzw. lediglich Aspekte wie die Gewichtung der unterschiedlichen Bestandteile des Sets zum Gegenstand partizipativer Verhandlungs- und Entscheidungsprozesse machen wollen (vgl. etwa Interview Wissenschaft o2). Für andere hingegen müsste dieses Indikatorenset selbst im Sinne einer „Vergemeinschaftung“ (Interview NGOs 03) zur Diskussion gestellt und im Rahmen „breite[r] BürgerInnenräte oder große[r] Beteiligungsverfahren“" (Interview NGOs O2) revidiert und solcherart "demokratisch komponiert“ (Interview NGOs 01) werden.
„Es liegt offenbar nicht an der Beamtenschaft, nicht an den Zahlen und auch nicht an der Statistik [...], warum die Implementierung von Indikatoren zur Beschreibung des gesellschaftlichen Wohlstandes so schwierig ist. Also ich glaube mittlerweile wirklich [...], man braucht ein neues Narrativ [...], ein neues Konzept und eine neue Theorie. [...] Man muss Geschichten erzählen, erklären, warum das von Relevanz ist. "(Interview Politik 01)

Problemformulierung I.3: Eine dritte Hürde im Bereich der breiten (medialen) Öffentlichkeit markiere schließlich eine Reihe von mit der medialen Situation verbundenen Problemen. Das betreffe etwa die Erscheinungsrhythmen von Berichten wie WGÖ? sowie die Medien und Formate ihrer Vermittlung (vgl. etwa Interviews Wissenschaft 01, Politik 05). Vor allem betreffe es jedoch die prekäre Lage des (Qualitäts-)Journalismus in Österreich (vgl. etwa Interviews Medien 01, Medien 02). Darüber hinaus sei ganz allgemein zu bedenken, dass nach einer lange anhaltenden Betrachtung von Fragen des Wohlstands und Fortschritts „durch diese Brille des BIP“ (Interview NGOs o3) ein Wandel Zeit benötige und wohl auch nur schrittweise vonstatten gehen werde (vgl. etwa Interviews Verbände 04, Politik 04, Verbände 03).

Lösungsansatz I.3: Die Überwindung der dritten Hürde wird in Einzelmaßnahmen wie kürzeren Intervallen der Berichterstattung oder einer Einbeziehung anderer Medien und Formate in die Vermittlung gesehen (vgl. etwa Interviews Wissenschaft o1, Politik 05). Darüber hinaus seien neue Kommunikations- und Medienstrategien zu entwickeln (vgl. etwa Interviews NGOs 02, Medien 01). Aber auch eine Veränderung der strukturellen Rahmenbedingungen des (Print-) Journalismus in Österreich gelte es zu überlegen (vgl. etwa Interviews NGOs 01, Medien 02). Zuvorderst gehe es jedoch um eine systematische, kontinuierliche und niederschwellige Form der Thematisierung (vgl. etwa Interviews NGOs 03, Verbände 04, Politik 01, Politik 02), zumal es sich bei der Entwicklung möglicher Alternativen zum BIP um ein - wie ein Medienvertreter meint - „großes Projekt [handelt], das lang braucht, um sich durchzusetzen“ (Interview Medien 02).

\subsection{Politisch-institutionelle Öffentlichkeit}

Problemformulierung II.1: Neben dem Umstand, „dass natürlich der Kapitalismus eine gewisse Eigenlogik“ (Interview Politik 02) habe und sich das Prinzip der Profitmaximierung in vielen Punkten mit einem erweiterten Wohlstands- und Fortschrittsverständnis 
als wenig kompatibel erweise, scheitere es im Bereich der politisch-institutionellen (Fach-)Öffentlichkeit zentral an divergierenden Interessenlagen und damit verbundenen Machtasymmetrien. Bereits die Frage alternativer Indikatoren, vor allem jedoch jene lebensqualitäts- und wohlstandsorientierter Politiken sei entsprechend Gegenstand politisch-ideologischer Kontroversen (vgl. etwa Interviews Politik 04, Politik o7). So werden etwa von ArbeitgeberInnenseite mit Blick auf Indikatorensets wie WGÖ? Aspekte wie Auswahl und Kohärenz hinsichtlich ihrer Ausgewogenheit und Konsensfähigkeit problematisiert (vgl. etwa Interviews Verbände 04, Verbände 05). Zudem unterbleibe bislang eine gezielte Akkordierung, häufig sogar eine klare Artikulation entsprechender Interessen, wie dies in Österreich ansonsten häufig über die mit der Sozialpartnerschaft assoziierten Gremien passiere (vgl. etwa Interviews Verbände 02, Verbände 03). Ein Experte aus dem Bereich Politik und Verwaltung meint dazu: „Es scheitert daran, dass es niemand fordert [...], und solange es keiner fordert, ist es auch kein Faktor in der Diskussion.“ (Interview Politik 03)

Lösungsansatz II.1: Die Überwindung dieser Hürde bestehe zentral darin, ausgehend von alternativen Indikatoren und ihrer Entwicklung politische Auseinandersetzungen über gesellschaftliche Ziele und damit verbundene Konflikte auszutragen (vgl. etwa Interviews Politik 02, Verbände 03). Dafür infrage kämen zivilgesellschaftliche und sozialpartnerschaftliche, vor allem jedoch parlamentarische Foren (vgl. etwa Interviews NGOs 02, Verbände o1, Wissenschaft 03, Politik 05). Dazu beitragen könne jedoch auch die Bundesregierung bspw. durch eine öffentliche Präsentation des WGÖ?-Berichts, wie dies etwa beim Wirtschaftsbericht Österreich, der alljährlich von mehreren Regierungsmitgliedern gemeinsam der Öffentlichkeit vorgestellt wird, heute bereits der Fall sei (vgl. etwa Interviews Verbände 03, Politik 04). In diesem Zusammenhang stelle sich auch die Frage, inwiefern eine gesetzliche Pflicht zur Beschäftigung mit bzw. zur Stellungnahme zu Berichten wie jenem der Statistik Austria verankert werden könne - und falls ja, für wen diese gelten solle (bspw. Bundesregierung oder BKA) (vgl. etwa Interview Politik 03).

Problemformulierung II.2: Eine weitere Hürde markiere die Art und Weise, wie gesellschaftlich dominante Vorstellungen von Wohlstand und Fortschritt sich auf staatlicher Ebene in bestimmte Institutionen (z.B. Fiskalrat) und Verfahren (z.B. Europäisches Semester) einschreiben und dadurch bestimmte Indikatoren
- unter austeritätspolitischen Vorzeichen etwa Kennziffern für Haushaltsdefizit und Verschuldung - auf Kosten anderer aufwerten (vgl. etwa Interviews Politik 03, Politik 06). Entscheidungen über gesellschaftliche Prioritätensetzungen, etwa vor dem Hintergrund von Trade-offs zwischen ökologischen und ökonomischen Zielen, würden so - auch aufgrund des Fehlens entsprechender institutioneller Mechanismen - der politisch-demokratischen Auseinandersetzung entzogen (vgl. etwa Interview Verbände 03). Dies gelte vor allem für mittel- und langfristig drängende Probleme, die durch den institutionell bedingten Fokus der Politik auf „tagesaktuelle Gegebenheiten“ (Interview Wissenschaft 03) weitgehend aus dem Blick geraten würden (vgl. etwa Interview Politik 02).

Lösungsansatz II.2: $\mathrm{Zu}$ überwinden wären diese Hürden aus der Perspektive der InterviewpartnerInnen, indem auf der Basis der oben dargelegten Auseinandersetzungen demokratische Entscheidungen über gesellschaftliche Prioritäten gefällt und diese institutionell festgeschrieben würden (vgl. etwa Interviews Verbände 03, Politik 03). Praktisch liefe dies - vergleichbar der Vorgehensweise im Rahmen von Europa 2020 - darauf hinaus, im Hinblick auf die Entwicklung der unterschiedlichen Indikatoren politisch $\mathrm{zu}$ erreichende Zielmarken $\mathrm{zu}$ setzen. In der Folge könnte die Politik auf das Erreichen solcher Ziele verpflichtet, der verfolgte Zielpfad in indikatorenbasierter Form kontrolliert und ggf. auch ein darauf bezogenes Bericht- und Sanktionswesen etabliert werden (vgl. etwa Interviews Politik 03, Politik 04). ${ }^{12}$ Von zentraler Bedeutung sei zudem eine „Legitimierung“ (Interview Politik 01) bestehender Initiativen, was etwa durch eine entsprechend, legitimierte' Person (z.B. Bundeskanzle-

12 Von manchen wird jedoch kritisiert, dass eine solche Orientierung am als ,neoliberal charakterisierten Konzept der Regelgebundenheit bestehende „Restriktionen für die Politik vergrößern“ (Interview Politik o3) würde. Diese Form der Institutionalisierung unterlaufe so tendenziell die Prinzipien repräsentativer Demokratie und sei deshalb als „technokratisch“ (Interview Politik o6) bzw. „expertokratisch“ (Interview Politik 07) zu problematisieren. Ein interviewter Politiker (Interview Politik o6) formuliert es drastisch: „, [R]egelgebundene Politik ist halt immer [...] das Misstrauen in die Politik, [...] ist im Ergebnis immer schlecht". Hinzu komme, dass politische Impacts - wie die praktische Irrelevanz der Europa-2020-Strategie exemplarisch vor Augen führe (vgl. etwa Interviews Politik 02, Verbände 01, NGOs 02, Verbände 02, Verbände 05) - auch im Falle einer solchen Form der Institutionalisierung nicht garantiert seien. 
rIn, MinisterIn) oder durch ein ebensolches Verfahren (z.B. EU-Verordnung, Ministerratsbeschluss) geleistet werden könne (vgl. etwa Interviews Politik 04, Politik 05).

Problemformulierung II.3: Als letzte Hürde lässt sich in diesem Bereich der Umstand bestimmen, dass das dominante Ressortdenken (vgl. etwa Interview Politik 06) sowie die mangelnde Initiative seitens des BKA als (potenziell) ressortübergreifende Instanz (vgl. etwa Interview Verbände 03) der Entwicklung eines holistischen Gesamtkonzepts entgegenstehe (vgl. etwa Interviews Wissenschaft 03, Politik 02). Ein solch integraler Ansatz wäre jedoch die Voraussetzung dafür, eine im sozial-ökologischen Sinn lebensqualitäts- und wohlstandsorientierte Politik verfolgen $\mathrm{zu}$ können. Dafür verantwortlich zeichne nicht zuletzt eine Form der institutionellen Trägheit (vgl. etwa Interview Verbände 04), zumal es laut einer Verbandsvertreterin „unglaublich schwer ist - gerade für politische Institutionen, die auch so eine Tradition haben -, eingetretene Pfade [...] zu verlassen“ (Interview Verbände o1). Relevant sei jedoch auch der Umstand, dass es im Bereich der wissenschaftlichen Politikberatung durch Beiräte bzw. Gremien an einschlägigen Einrichtungen mit einem solch weit gefassten Auftrag fehle und es in bestehenden Einrichtungen wie dem Fiskalrat diesbezüglich wenig Spielraum gebe (vgl. etwa Interviews Politik 03, Verbände 03).

Lösungsansatz II.3: Die Überwindung dieser letzten Hürde wird in einer „ressortübergreifenden Koordination" (Interview Wissenschaft 03) durch das BKA bzw. in anderen Formen einer interministeriellen Zusammenarbeit gesehen (vgl. etwa Interview Verbände 03). Dies könnte - in Anlehnung an den Wirtschaftsbericht Österreich - im Rahmen eines jährlich erscheinenden Wohlstandsberichts geschehen, der etwa als ressortübergreifendes Projekt durch die Verwaltung zu erstellen wäre (vgl. Interview Politik 04), was auch eine Sensibilisierung der BeamtInnenschaft ermöglichen würde (vgl. etwa Interviews Politik 02, Politik 05). Darüber soll es - etwa unter Bezugnahme auf die Debatte um ein ,magisches Vier- bzw. Vieleck der Wirtschaftspolitik' (vgl. etwa Interviews Verbände o3, Politik 05) - gelingen, ein konsistentes Gesamtkonzept für eine lebensqualitäts- und wohlstandsorientierte Politik zu erarbeiten. Dessen Durchsetzung wiederum könne - angesichts von pfadabhängiger Trägheit und damit verbundenem Konservatismus - am ehesten mittels der Formulierung einer umfassenden Vision gelingen (vgl. etwa Interviews Verbände o1, Verbände
02). Im Bereich der Politikberatung solle dies durch ein eigenes ExpertInnengremium etwa in Gestalt eines Wohlstandsrats unterstützt werden, das aus dem WGÖ?-Bericht Schlussfolgerungen abzuleiten und die Regierung zu beraten hätte (vgl. etwa Interviews Verbände 03 , Wissenschaft 03$)$. $^{13}$

\section{Schlussfolgerungen: Restriktionen reflektieren und überwinden}

Auch in Österreich hat die Krise ab 2007 ein Möglichkeitsfenster geöffnet, wodurch die einseitig wachstumsorientierte Wirtschaftspolitik der vorangegangenen Jahre und Jahrzehnte zumindest kurzzeitig an prominenter Stelle als Problem auf der politischen Agenda erschien bzw. auf der politischen Prioritätenliste nach oben rückte. ${ }^{14}$ Vor dem Hintergrund der parallel dazu auf internationaler Ebene initiierten Debatten um alternative Indikatoren gesellschaftlichen Fortschritts und Wohlstands veränderten sich in der Folge die Möglichkeiten eines darauf bezogenen Agenda-Settings. Konkret ergab sich vor allem mit Blick auf soziale, in abgeschwächter Form aber auch auf ökologische Ziele die Gelegenheit, lebensqualitätsund wohlstandsorientierte Ansätze als Lösungen für das Problem verkürzter Wirtschaftspolitik ins Spiel zu bringen und Interessenkoalitionen für eine Unterstützung solcher Ansätze zu schmieden. Im Anschluss an Kingdon (1984: 182) kann vor dem Hintergrund der Öffnung eines sogenannten "problem window“ von der Möglichkeit gesprochen werden, Politics und Policies Streams mit dem Problem Stream zu verkoppeln, um solcherart auf einen grundlegenden Policy Change hinzuwirken. Eine zentrale - und von Kingdon weit-

13 Auch dieser Vorschlag wird vereinzelt problematisiert, da im Rahmen solcher Gremien die Politik den im Kontext repräsentativer Demokratie eigentlich dafür Verantwortlichen entrissen und „,an irgendein Board von Wissenschaftlern" (Interview Politik 03) ausgelagert werde.

14 Relativierend ist jedoch anzumerken, dass die Krise im Kontext der Wachstums- und Indikatorendebatte ambivalente Effekte zeitigte. Schließlich war diese nicht bloß mit der Chance zu einer an alternativen Indikatoren orientierten Neuausrichtung der Wirtschaftspolitik verbunden, sondern auch mit der Gefahr einer an traditionellen Indikatoren (Wachstum, Beschäftigung) orientierten Stärkung ihrer bisherigen Ausrichtung (vgl. etwa Bleys/Whitby 2015: 166; Fioramonti 2013: 154f.; Jackson 2013: 16ff.). Ob das - angesichts positiver Wachstums- und Beschäftigungsprognosen - aktuell häufig konstatierte ,Ende der Krise' die angesprochenen Chancen für eine Neuausrichtung verbessern wird, ist freilich offen. 
Tabelle 3: Beschränkungen für eine Verankerung wohlstandsorientierter Politik

\begin{tabular}{|l|l|}
\hline Endogene Restriktionen & Formen diskursiver Pfadabhängigkeit \\
\hline & $\begin{array}{l}\text { (1) Politische Inhalte: Pfadabhängige Rezeption programmatischer Ideen in Bezug } \\
\text { auf Wohlstand/Lebensqualität innerhalb des vom wachstumsorientierten Policy- } \\
\text { Paradigma abgesteckten ,Korridors' (z.B. Ergänzung des BIP durch alternative } \\
\text { Indikatoren statt Ersetzung durch ein alternatives Wohlstandsmaß). }\end{array}$ \\
\hline $\begin{array}{l}\text { (2) Formen von Politik: Pfadabhängige Konzeptualisierung lebensqualitäts- und } \\
\text { wohlstandsorientierter Politik innerhalb dieses ,Korridors' statt Einbindung ,neuer' } \\
\text { (Kollektiv-)Akteure (z.B. NGOs, Bewegungsinitiativen) bzw. Entwicklung ,neuer' } \\
\text { Formate (z.B. Beteiligungsverfahren, Kampagnen). }\end{array}$ \\
\hline Exogene Restriktionen & \begin{tabular}{l} 
Formen epistemischer Selektivität \\
\hline (3) Kräfteverhältnisse: Beschränkung jenes Bereichs, über den politisch \\
entschieden wird, aufgrund interessengeleiteter Formen der Filterung bzw. des \\
Ausschlusses bestimmter Problemperzeptionen bzw. darauf bezogener \\
Lösungsansätze (z.B. auf Verbindlichkeit basierende Ansätze).
\end{tabular} \\
& $\begin{array}{l}\text { (4) Institutionengefüge: } \text { Mit dem institutionellen Aufbau des Staates (z.B. } \\
\text { Ressortprinzip) bzw. mit konkreten Institutionen (z.B. Fiskalrat) und Verfahren } \\
\text { (z.B. Europäisches Semester) verbundene Form der Filterung bzw. des } \\
\text { Ausschlusses (relativ unabhängig von momentanen Kräfteverhältnissen). }\end{array}$ \\
\hline
\end{tabular}

Quelle: Eigene Darstellung

gehend ignorierte (vgl. Bache 2013: 22/29ff.; Bache/ Reardon 2013: 898/907f., 2016: 14ff./145ff.) - Rolle kam dabei der Entwicklung und Diffusion entsprechender Ideen über internationale Policy-Netzwerke bzw. transnationale Policy-Communitys zu (vgl. ebd.).

In Österreich traten in diesem Kontext an der Schnittstelle von Wissenschaft und Verwaltung - konkret etwa in der Statistik Austria, aber auch am WIFO oder im Bundesministerium für Land- und Forstwirtschaft, Umwelt und Wasserwirtschaft (BMLFUW) Policy Entrepreneurs ${ }^{15}$ auf den Plan, um das dargestellte Möglichkeitsfenster für eine Stärkung von Ansätzen lebensqualitäts- und wohlstandsorientierter Politik zu nutzen. Im Rahmen von Projekten wie WGÖ?, dem Forschungsprojekt WWWforEurope $e^{16}$ oder auch der Initiative Wachstum im Wandel ${ }^{17}$ wurden von den angesprochenen AkteurInnen erhebliche Ressourcen (u.a. Geld, Reputation) in dieses Vorhaben investiert.

Was die Frames bzw. Deutungsmuster anbelangt, mit deren Hilfe das politische Sachthema diskursiv gerahmt wurde, bemühte man sich vor allem im Zusammenhang mit den beiden letztgenannten Projekten darum, unter Rekurs auf die Indikatorendebatte ein erweitertes Wohlstandsverständnis zu entwickeln

15 Mitunter ist im vorliegenden Kontext in diesem Sinn auch von „indicator entrepreneurs" bzw. „statistical entrepreneurs" (Whitby et al. 2014: 40; Bleys/Whitby 2015: 169/170) die Rede.

16 Vgl. dazu www.foreurope.eu (31.3.2017).

17 Vgl. dazu www.wachstumimwandel.at (31.3.2017). und zugleich darauf bezogene (u.a. ressortübergreifende) Interessenkoalitionen zu schmieden. Kingdon (1984: 4) folgend gelang es darüber zwar, das Thema auf die Governmental Agenda zu setzen und - vor allem bei einzelnen Stakeholdern und in den mit diesen verbundenen (Teil-)Öffentlichkeiten - Aufmerksamkeit zu generieren. Als entscheidungsrelevante Materie auf die Decision Agenda bzw. auf die oberen Ränge der politischen Prioritätenliste schaffte es das Thema jedoch nicht.

Forschungsarbeiten $\mathrm{zu}$ anderen Ländern mit vergleichbarem Design kommen zu ähnlichen Ergebnissen und sehen den Grund hierfür in einer unzureichenden Koppelung des Problem-Streams aufgrund eines misslungenen Framings (vgl. Bache 2013: 24/31ff.; Bache/Reardon 2013: 900/909f.). Auch unsere Untersuchung legt nahe, dass es bislang weitgehend misslungen ist, lebensqualitäts- und wohlstandsorientierte Politik (sowie damit verbundene Interessenkoalitionen) als konkrete Lösung auf das Problem einer einseitig verkürzten Wirtschaftspolitik zu beziehen und in diesem Sinn den Problem-Stream mit den Policies- und PoliticsStreams zu verkoppeln. Wenngleich die Durchsetzung entsprechender Frames in den damit verbundenen Deutungskämpfen als Voraussetzung einer solchen Koppelung gelten kann, ist unseres Erachtens damit allerdings noch nicht erklärt, warum dies bislang misslingt. Wir unterstreichen daher nochmals die Bedeutung der unterschiedlichen endogenen bzw. exogenen Beschränkungen und Hindernisse (vgl. Tabelle III). 
5.1 Endogene Restriktionen: Formen diskursiver Pfadabhängigkeit

Was die endogenen Restriktionen anbelangt, geht es aufgrund des derzeit hegemonialen Policy-Paradigmas darum, dass vor dem Hintergrund institutionell sedimentierter Wissensbestände und eines entsprechend variierenden ,Matchings' bestimmte politische Konzepte als passfähiger gelten, weil sie mit besagten Wissensbeständen in Kontinuität stehen und deshalb im Sinne pfadabhängiger Entwicklung gegenüber anderen priorisiert werden.

Eine so verstandene diskursive Pfadabhängigkeit drückt sich (1) inhaltlich in der Schwierigkeit aus, neue programmatische Ideen in Bezug auf Wohlstand und Lebensqualität aufzunehmen und im Sinne des damit verbundenen Paradigmenwechsels politisch umzusetzen. Im Kontext der neueren Indikatorendebatte lässt sich dies exemplarisch an einer frühzeitig gefällten Richtungsentscheidung verdeutlichen: Im Ansatz bereits im SSF-Report (vgl. Stiglitz et al. 2009: u.a. 77) stark gemacht und von späteren Berichten (vgl. etwa SVR/ CAE 2010: 1f./28) bestätigt wird nämlich eine Präferenz für Dashboard- gegenüber Composite-Indicator-Ansätzen, also für Indikatorensets gegenüber zusammengesetzten Indikatoren (vgl. etwa Pesendorfer/Leitner 2010: 536ff.). In der Praxis lief dies - wie es etwa auch bei $W G O ̈$ ? der Fall ist (Stichwort: ,Indikatorenset BIP + $\left.30^{\circ}\right)$ - darauf hinaus, das BIP durch alternative Indikatoren zu ergänzen anstatt es durch ein alternatives Wohlstandsmaß im Sinne einer neuen Single Magic Number zu ersetzen. Dagegen formulierte Einwände (vgl. etwa Constanza et al. 2016: 351; Diefenbacher/Zieschank 2011: 59) ebenso wie der damit verbundene Ruf „to dethrone GDP“" (Constanza et al. 2014: 283; Fioramonti 2013: 83) wurden dem gegenüber marginalisiert.

Zweifelsohne war eine solche, pfadabhängige Lösung', wie sie auf EU-Ebene in der bezeichnenden Verschiebung von Beyond GDP zu GDP and Beyond zum Ausdruck kam, einfacher umsetzbar. Zugleich scheint sich jedoch die mit Dashboard-Ansätzen verbundene Befürchtung zu bestätigen, dass der eigentlich intendierte Wandel ausbleibt, das mit dem BIP-Wachstum verbundene Policy-Paradigma unhinterfragt fortbesteht - und in der Konsequenz die alternativen Indikatoren als letztlich nebensächliches Beiwerk zur eigentlich relevanten Kennzahl des BIP wahrgenommen werden (vgl. ebd.: 102f./113ff.): „At best they constitute a side dish', while the media hunger is all focused on GDP as the main course." (ebd.: 115)
In den Interviews drückte sich diese inhaltliche Dimension der diskursiven Pfadabhängigkeit so auch darin aus, dass - trotz der verbreiteten Skepsis gegenüber der Vermittelbarkeit von mit Dashboard-Ansätzen verbundenen Indikatorensets - die Möglichkeit der Etablierung eines alternativen Wohlstandsmaßes in Gestalt eines Composite Indicators von wenigen Ausnahmen abgesehen (vgl. etwa Interview Medien o2) keine Erwähnung fand. Unabhängig davon, was aus fachlicher Perspektive jeweils für oder gegen diese Ansätze sprechen mag, kann dies doch als Indiz dafür dienen, wie wenig der vom wachstumsorientierten PolicyParadigma abgesteckte ,Korridor' bislang theoretisch hinterfragt oder gar praktisch überschritten wird.

Besagte Pfadabhängigkeit zeigt sich (2) in formaler Hinsicht etwa darin, dass eine Einbindung ,neuer - jenseits des ,traditionellen' Felds lokalisierter - (Kollektiv-)Akteure (z.B. NGOs, BürgerInneninitiativen) ebenso wie ein Beschreiten ,neuer - über ,traditionelle Kanäle und Formate hinausgehender - Wege (z.B. Beteiligungsverfahren, Kampagnen) hierzulande bislang schwerfällt. Auch im progressiven Lager bleibt die Vorstellung von Politik hinsichtlich ihrer Formbestimmtheit daher weitgehend auf Akteure und Formate beschränkt, die eng mit dem Wachstumsparadigma in der Wirtschaftspolitik assoziiert sind. Dies gilt für Akteure aus den Bereichen Politik/Verwaltung, Verbände (Sozialpartner) und Wissenschaft sowie für Formate, die entweder traditionell bestimmend waren (z.B. Beirat für Wirtschafts- und Sozialfragen) oder die im Zuge der neoliberalen Integrationsweise der EU verankert wurden (z.B. ExpertInnenräte). Der in der internationalen Debatte häufige Hinweis auf die Notwendigkeit eines „,bottom-up engagement of civil society" (Constanza et al. 2014: 285) oder gar eines von Bewegungsinitiativen aus dem Kontext der Transitionbzw. Degrowth-Bewegung anzustoßenden „change from below" (vgl. Fioramonti 2013: 119ff.; Brand 2014) findet hingegen wenig Beachtung. ${ }^{18}$ Dasselbe gilt für

18 Wie in Studien zum Thema betont wird, könne es in diesem Zusammenhang kaum überraschen, dass es „little evidence of strong, bottom up' societal demand for indicators themselves" (Whitby et al. 2014: 18) gebe, wurde die Indikatorendebatte doch lange Zeit nahezu exklusiv von StatistikerInnen und ÖkonomInnen geführt. In vermittelter Form wie etwa in Gestalt von Forderungen nach einem sozial-ökologischen Wandel seien diese aber weit verbreitet - und entsprechend sei die Zivilgesellschaft auch verstärkt in Debatten um die Bedeutung von gesellschaftlichem Fortschritt und damit assoziierten Indikatoren einzubinden (ebd.: 36). 
neue Formate von Politik, die eine politische Partizipation und soziale Teilhabe der genannten Akteure erst ermöglichen würden.

Dieser formale Aspekt diskursiver Pfadabhängigkeit drückte sich in den Interviews etwa darin aus, dass trotz des vielfach artikulierten Problembewusstseins bezüglich der mangelhaften Verankerung des Themas in der breiten Öffentlichkeit solche,neuen' Akteure bzw. Formate in den Überlegungen der InterviewpartnerInnen - jenseits der einschlägig damit befassten Kreise (vgl. etwa Interviews NGOs o1, NGOs 02) - weitgehend außen vor blieben. ${ }^{19}$ Eine Ausnahme markierte in diesem Fall ein Vertreter des WIFO (Interview Wissenschaft 02). Entsprechende Vorschläge fanden so auch in dem vom WIFO koordinierten Forschungsprojekt WWWforEurope ihren Niederschlag. Konkret wird im Hinblick auf die intendierte „sozio-ökologische Transition“ (Aiginger 2016: 55) hier etwa über die Integration neuer (zivilgesellschaftlicher) Akteure (z.B. NGOs oder Jugend- und Alternativgruppen) als mögliche Vermittler des Wandels nachgedacht, um darüber eine den Wandel befördernde Kultur zu kreieren (ebd.: 108ff.). ${ }^{20}$

\subsection{Exogene Restriktionen: Formen epistemi- scher Selektivität}

Was schließlich die exogenen Restriktionen betrifft, geht es um jene ,epistemischen Selektivitäten' politischer Institutionen, die im Sinne vielfältiger Filterungsund Ausschlussmechanismen eine Beschränkung des politischen Entscheidungsbereichs auf spezifische Lösungsvorschläge bzw. damit verbundene Problemwahrnehmungen begünstigen.

Dem Agenda-Setting für eine lebensqualitäts- und wohlstandsorientierte Politik entgegen stehen in diesem Fall (3) Hürden, die aufgrund der gesellschaftlichen

19 Besonders augenfällig ist dies im Zusammenhang mit dem „breiten Kommunikationsprozess“ (Statistik Austria 2015: 31), der - entsprechenden Empfehlungen auf internationaler Ebene folgend (vgl. Stiglitz et al. 2009: 18; ESSC 2011: 10) - im Rahmen von WGÖ? etabliert wurde. Auch in diese Stakeholder-Dialoge wurden und werden nämlich lediglich die genannten ,traditionellen' Akteure aus Wissenschaft, Verwaltung und Verbänden einbezogen (Pesendorfer et al. 2012: $7 \mathrm{f} . / 19)$.

20 Dabei geht es vor allem um die von solchen (Kollektiv-)Akteuren im Rahmen zivilgesellschaftlicher Bottomup-Initiativen kreierten Praktiken und Arrangements (z.B. Sharing Economy, Circular Economy) verstanden als „laboratories for a more sustainable way to produce and to consume" (Aiginger 2016: 114).
Interessenkonstellationen und Kräfteverhältnisse den Bereich dessen, worüber politisch entschieden wird, restringieren. Evident ist dies vor allem im Zusammenhang mit Fragen, bei denen die allgemeine Zustimmung zu Well-Being als ,Wohlfühlthema', „wo man möglichst viele in ein Boot bekommt" (Interview NGOs 02), an ihre Grenzen stößt. Dies zeigt sich etwa, wenn im Hinblick auf die Entwicklung bestimmter Indikatoren (z.B. Treibhausgasemissionen; Vermögensverteilung) verbindliche Zielmarken (z.B. Reduktionsziele) formuliert und damit verbundene Maßnahmen (z.B. Umweltauflagen; Vermögenssteuern) ergriffen werden sollen.

Von ArbeitgeberInnenseite wurden in den Interviews trotz grundsätzlicher Zustimmung zu Ansätzen lebensqualitäts- und wohlstandsorientierter Politik entsprechend alle Strategien infrage gestellt, die auf deren bindenden Charakter setzen: „[D]ie Forderungen nach einem Beyond GDP [ist] eine legitime Forderung [...], die - glaube ich - jeder von uns teilen würde. Die Frage ist dann, inwieweit ist diese Ausweitung der Indikatoren [...] eine Hilfestellung für die Anwender oder inwiefern ist sie bindend“ (Interview Verbände 05). Eine „hoheitliche Bindung“ (Interview Verbände o4) lehnten die interviewten Vertreter von Industriellenvereinigung (IV) und Wirtschaftskammer (WKO) dabei gleichermaßen ab. ${ }^{21}$ Vor diesem Hintergrund verwundert es wenig, dass das Spektrum möglicher Lösungsvorschläge bzw. damit verbundener Problemperzeptionen bislang stark auf Kosten von Ansätzen beschränkt scheint, die auf Verbindlichkeit insistieren. Das heißt, deren Durchsetzungschancen sind angesichts der epistemischen Selektivität staatlicher Institutionen insofern limitiert, als sie erst gar nicht zum Gegenstand politischer Entscheidungen werden. ${ }^{22}$

Schließlich kann (4) auch die Art und Weise, wie dominante Vorstellungen gesellschaftlichen Wohlstands und Fortschritts in das staatliche Institutionengefüge eingeschrieben sind, die Filterung und den Ausschluss alternativer Ansätze und damit verbunde-

21 Vor allem im sozialen Bereich dementiert wird dadurch auch das im Kontext von Debatten um Wohlstand und Lebensqualität häufig getätigte Postulat, demzufolge das Thema "transcends traditional left-right cleavages" (Bache 2013: 35; Bache/Reardon 2013: 911).

22 Wie in den Interviews betont wird (vgl. etwa Interviews Wissenschaft 03, Politik 01), ist dies bereits im Bereich des Sozialen evident, gilt aber insofern noch mehr im Bereich der Ökologie, als es hier keine - den gesetzlichen Interessenvertretungen von Arbeit und Kapital vergleichbare - Repräsentanz von Interessen gibt. 
ner Wissensformen erklären. Wie erwähnt, zeigt sich dies bereits an Institutionen wie dem Fiskalrat und Verfahren wie dem Europäischen Semester, in denen sich die aktuelle wirtschaftspolitische Orientierung an Prinzipien der Austerität und Wettbewerbsfähigkeit institutionell manifestiert. Dies erklärt, wie auch in den Interviews betont wurde (vgl. etwa Interviews Politik 03, Politik 06), warum formal gleichrangige Indikatoren real durch ein starkes Ungleichgewicht hinsichtlich ihres politischen Impacts gekennzeichnet sind, ohne dass dies das Zutun politischer Akteure bedürfte oder durch eine Verschiebung der politischen Kräfteverhältnisse automatisch verändert würde. Auf einer grundlegenderen Ebene gilt dies in analoger Form für die Wachstumsorientierung als solche, seien doch - nicht zuletzt aufgrund der vermeintlichen Objektivität und Ideologiefreiheit dieser Kennziffer (Lepenies 2013: 183) - „das ganze Staatssystem und alle Berichte auf dem BIP aufgebaut“ (Interview Politik 07). Das damit verbundene Wachstumsparadigma ist entsprechend tief im politischen System bzw. seiner verbandsförmigen Erweiterung in Gestalt der Sozialpartnerschaft verankert. Lebensqualitäts- und wohlstandsorientierte Politik verstanden als „an integrated policy process that also informs a more holistic approach to economic policy making" (Whitby et al. 2014: 3/12) sowie damit verbundene Wissensformen sind so mit Formen epistemischer Selektivität konfrontiert, die unmittelbar mit dem institutionellen Aufbau des Staates (z.B. Ressortprinzip) in Beziehung stehen (ebd.: 31ff.; Bleys/Whitby 2015: 168).

\section{Schluss und offene Fragen}

Das österreichische Wirtschaftsministerium bemüht auf seiner Website eine Definition von Wirtschaftspolitik, die deutlich von der Debatte um eine sozial-ökologische Erweiterung des dominanten Fortschritts- und Wohlstandsverständnisses beeinflusst ist: Ihr Ziel bestehe nämlich „in einer Erhöhung des Wohlstands und in einer nachhaltigen Verbesserung der Lebensqualität der Bürger [...] unter Berücksichtigung angemessener Sozial- und Umweltstandards “ ${ }^{23}$ Jenseits dieser Definition ist von einem solcherart erweiterten Verständnis freilich wenig $\mathrm{zu}$ erahnen. Rund zehn Jahre nach dem Ausbruch der Finanz- und Wirtschaftskrise und nach dem Beginn der sie begleitenden Wachstums- und Indikatorendebatte ist in der öster-

23 www.bmwfw.gv.at/Wirtschaftspolitik/Wirtschaftspolitik/Seiten/default.aspx (31.03.2017). reichischen Wirtschaftspolitik vielmehr - von einigen rhetorischen Blüten abgesehen - ,alles beim Alten'

Wie aus den in diesem Artikel dargelegten Entwicklungen ersichtlich wird, hat die Diskussion um den Stellenwert des BIP jedoch einen gesellschaftspolitischen Kern: Die Art und Weise, wie Wohlstand geschaffen wird - d.h. wie die Bedingungen für ein auskömmliches und sinnerfülltes Leben in einer Gesellschaft gestaltet sind -, ist von historischen Erfahrungen geprägt, wie sie etwa während des, goldenen Zeitalters des Kapitalismus' nach dem Zweiten Weltkrieg gemacht wurden. Vor allem jedoch hängt sie von politischen Auseinandersetzungen und Strategien ab; das heißt, sie ist umkämpft. Das impliziert, dass auch die Frage, inwiefern Versuche zur Lösung der aktuellen Krise Teil einer kohärent formulierten Wirtschafts- und Gesellschaftspolitik sind oder eher Teil eines krisengetriebenen und durch die Positionssicherung der Eliten induzierten, muddling through', historisch offen ist.

Um in diesem Sinn Ansätze einer lebensqualitätsund wohlstandsorientierten Politik in Österreich an zentraler Stelle auf die politische Agenda $\mathrm{zu}$ setzen, wird es nicht zuletzt darum gehen, die dargestellten endogenen bzw. exogenen Restriktionen abzumildern bzw. zu neutralisieren. Dabei müssen zum einen Strategien entwickelt werden, um die aus den gesellschaftlichen Kräfteverhältnissen resultierenden Widerstände gegen Veränderungen zu überwinden. Oder wie Whitby et al. (2014: 39) es formulieren: „[A]dvocates of change must have a strategy for neutralising that resistance“. Darüber hinaus braucht es jedoch auch Strategien für einen grundlegenderen Umbau des staatlichen Institutionengefüges als Voraussetzung für eine effektive Außerkraftsetzung bestehender Filterungs- und Ausschlussmechanismen im Sinne der epistemischen Selektivität. Zugleich gilt es, Strategien für eine Überschreitung jenes ,Korridors' auszuarbeiten, der im Sinne diskursiver Pfadabhängigkeit durch das dominante wachstumsorientierte Policy-Paradigma abgesteckt wird. In inhaltlicher Hinsicht könnte dies auf die Förderung eines neuen Wohlstandsmaßes als Alternative zum BIP hinauslaufen; in formaler Hinsicht auf eine Stärkung neuer Formen von Politik in Abgrenzung zu den mit dem Wachstumsparadigma verbundenen Formaten und Akteuren bzw. auf eine Umarbeitung der Interessen Letzterer.

Von zentraler Bedeutung dürfte dabei die Formulierung eines kohärenten und überzeugenden (Meta-) Narrativs sein, dessen Fehlen auch in der Literatur immer wieder bemängelt wird (vgl. indikatorenzent- 
riert etwa Bleys/Whitby 2015: 169; Whitby et al. 2014: 28/36f.; gesellschaftspolitisch etwa Thie 2013). Wie von mehreren InterviewpartnerInnen betont, müsste ein alternatives Wohlstandsmaß dabei insofern vergleichbar dem BIP gerahmt und vermittelt werden, als dessen unmittelbare Relevanz und praktische Bedeutung - im Sinne von: ,Wenn es wächst, geht's uns besser!' - sich auch jenen erschließe, die weder um die Kennziffer selbst noch um deren Ausmaß wissen (vgl. etwa Interview Politik 01). Als ein erster Schritt der Annäherung an ein solches Narrativ ließe sich dabei das, Gute Leben für alle als Fokus - und die Infragestellung aktuell dominanter Produktions- und Lebensweisen als Voraussetzung - bestimmen (Brand/Wissen 2017: 165ff.). ${ }^{24}$

Die Vertiefung der hier präsentierten Untersuchung würde von international vergleichenden Studien profitieren, da diese Diskussionen auch in anderen Ländern geführt werden. Wir wollen abschließend jedoch ein Problem benennen, für das es keine einfache Lösung gibt: Lebensqualitäts- und wohlstandsorientierte Politik jenseits kapitalistischer Wachstumsimperative und dem Diktum der Wettbewerbsfähigkeit wird, wenn überhaupt, aktuell weitgehend auf nationalstaatlicher Ebene, allenfalls noch auf jener der Europäischen Union, gedacht. Ausdruck dessen sind nicht nur die statistischen Apparate, sondern vor allem die Struktur und Legitimation von Politik selbst. Auch sozial-ökologischer Wohlstand, der Fragen von sinnvoller Produktion und ebensolchem Konsum, von sozialer und ökologischer Gerechtigkeit und Ähnliches impliziert, scheint allzu oft im Rahmen nationalstaatlich verfasster Gesellschaften gedacht und gemacht zu werden. Wie aber können zum Ersten die Voraussetzungen einer als problematisch erachteten, einseitig auf Wirtschaftswachstum setzenden ,imperialen Produktions- und Lebensweise in den Blick geraten? Dabei geht es etwa um die billigen Arbeitskräfte und Ressourcen, die andernorts genutzt werden, um in einem Land wie Österreich materiellen Wohlstand $\mathrm{zu}$ erzeugen. Und zweitens: Wie kann sozial-ökologischer Wohlstand jenseits nationalstaatlich verfasster Grenzen gedacht und realisiert werden, nämlich tendenziell wirklich ,für alle'? Um diese Fragen kommt auch die progressive Debatte um (Post-)Wachstum, Wohlstand und Lebensqualität nicht herum.

24 Vgl. in diesem Sinn auch www.guteslebenfueralle. org (31.03.2017).

\section{Literatur}

Aiginger, K. (2016): New Dynamics for Europe: Reaping the Benefits of Socio-ecological Transition. WWWforEurope Synthesis Report Part I. Wien: WIFO.

Bachrach, P./Baratz, M. S. (1975/1970): Zwei Gesichter der Macht. In: Narr, W.-D./Offe, C. (Hg.): Wohlfahrtsstaat und Massenloyalität. Köln: Kiepenheuer und Witsch, 224-234.

Bache, I. (2013): Measuring quality of life for public policy: an idea whose time has come? Agenda-setting dynamics in the European Union. Journal of European Public Policy, $20(1), 21-38$.

Bache, I./Reardon L. (2013): An Idea Whose Time has Come? Explaining the Rise of Well-Being in British Politics. Political Studies, 61 (4), 898-914.

Bache, I./Reardon L. (2016): The Politics and Policy of Wellbeing: Understanding the Rise and Significance of a New Agenda. Cheltenham: Edward Elgar.

Bleys, B./Whitby, A. (2015): Barriers and opportunities for alternative measures of economic welfare. Ecological Economics, 117, 162-172.

Bogner, A./Littig, B./Menz, W. (2014): Interviews mit Experten. Eine praxisorientierte Einführung. Wiesbaden: Springer VS.

Brand, U. (2013): State, context and correspondence. Contours of a historical-materialist policy analysis. Österreichische Zeitschrift für Politikwissenschaft, 42 (4), 425-442.

Brand, U. (2014): Kapitalistisches Wachstum und soziale Herrschaft. Motive, Argumente und Schwächen grundlegender Wachstumskritik. Prokla 175, 44 (2), 289-306.

Brand, U./Vadrot, A. (2013): Epistemic Selectivities and the Valorisation of Nature: The Cases of the Nagoya Protocol and the Intergovernmental Science-Policy Platform for Biodiversity and Ecosystem Services (IPBES). Law, Environment and Development Journal, 9 (2), 204-220.

Brand, U./Wissen, M. (2017): Imperiale Lebensweise. Zur Ausbeutung von Mensch und Natur in Zeiten des globalen Kapitalismus. München: Oekom Verlag.

Brangsch, L. (2011): Kennziffernfragen sind Machtfragen. Politische Dimensionen von Gegenstand und Methode von Wachstumsdebatten. Kurswechsel, 1/2011, 25-38.

Chaloupek, G./Feigl, G. (2012): Die Wachstumskontroverse vor vierzig Jahren und heute. Wirtschaft und Gesellschaft, 38 (4), 771-80o.

Costanza, R./Giovannini, E./Kubiszewski, I./Lovins, H./ McGlade, J./Pickett, K. E./Ragnarsdottir, K. V./ Roberts, D./De Vogli, R./Wilkinson, R. (2014): Development: Time to leave GDP behind. Nature, Vol. 505 (7483), 283285.

Costanza, R./Daly, L./Fioramonti, L./Giovannini, E./Kubiszewski, I./Mortensen, L. F./Pickett, K. E./Ragnarsdottir, K. V./De Vogli, R./Wilkinson, R. (2016): Modelling and measuring sustainable wellbeing in connection with the UN Sustainable Development Goals. Ecological Economics, $130,350-355$. 
Demirović, A./Dück, J./Becker, F./Bader, P. (Hg.) (2011): Vielfachkrise im finanzdominierten Kapitalismus. Hamburg: VSA.

Deutscher Bundestag (2013): Schlussbericht der Enquete-Kommission „Wachstum, Wohlstand, Lebensqualität - Wege zu nachhaltigem Wirtschaften und gesellschaftlichem Fortschritt in der Sozialen Marktwirtschaft". Drucksache 17/13300. Berlin.

Diefenbacher, H./Zieschank, R. (2011): Woran sich Wohlstand wirklich messen lässt. Alternativen zum Bruttoinlandsprodukt. München: Oekom Verlag.

Dullien, S./Van Treeck, T. (2012): Ziele und Zielkonflikte der Wirtschaftspolitik und Ansätze für Indikatoren und Politikberatung. IMK Policy Brief. Düsseldorf.

Europäische Kommission (2009): GDP and beyond. Measuring progress in a changing world. $\mathrm{COM(2009)} 433$ final. Brüssel.

Europäische Kommission (2013): Progress on „GDP and beyond" actions. SWD(2013) 303 final. Brüssel.

Europäische Kommission (2014): Bestandsaufnahme der Strategie Europa 2020 für intelligentes, nachhaltiges und integratives Wachstum. COM(2014) 130. Brüssel.

Europäisches Parlament (2011): GDP and beyond - Measuring progress in a changing world. P7_TA(2011)0264. Brüssel.

ESSC. European Statistical System Committee (2011): Sponsorship Group on Measuring Progress, Well-being and Sustainable Development. Final Report. Brüssel: ESSC.

Feigl, G. (2017): Von der Neuvermessung gesellschaftlichen Wohlstands zur wohlstandsorientierten Wirtschaftspolitik. Momentum Quarterly, 6 (2), 138-152.

Fioramonti, L. (2013): Gross Domestic Problem: The Politics Behind the World's Most Powerful Number. London/New York: Zed Books.

Fischer, F./Gottweis, H. (Hg.) (2012): The Argumentative Turn Revisited. Durham: Duke University Press.

Gläser, J./Laudel, G. (2010): Experteninterviews und qualitative Inhaltsanalyse. Wiesbaden: VS Verlag.

Gramsci, A. (1991ff./1948ff.): Gefängnishefte. Kritische Gesamtausgabe. Hamburg: Argument.

Griesser, M./Brand, U. (2016): Verankerung wohlstandorientierter Politik. Working Paper der Kammer für Arbeiter und Angestellte für Wien, Reihe „Materialien zu Wirtschaft und Gesellschaft", Nr. 165. Wien.

Griesser, M. (2017): Images and imaginaries of unemployed people. Discursive shifts in the transition from active to activating labour market policies in Germany. Critical Social Policy [online first], 1-20.

Hall, P. A. (1993): Policy Paradigms, Social Learning, and the State. The Case of Economic Policymaking in Britain. Comparative Politics, 25 (3), 275-296.

Jackson, T. (2013/2009): Wohlstand ohne Wachstum: Leben und Wirtschaften in einer endlichen Welt. München: Oekom Verlag.

Jessop, B. (2002): The Future of the Capitalist State. Cambridge/Malden: Polity.
Kingdon, J. W. (1984): Agendas, Alternatives, and Public Policies. New York: Harper Collins Publishers.

Knecht, A. (2010): Lebensqualität produzieren. Ressourcentheorie und Machtanalyse des Wohlfahrtsstaats. Wiesbaden: VS-Verlag.

Lepenies, P. (2013): Die Macht der einen Zahl. Eine politische Geschichte des Bruttoinlandsprodukts. Berlin: Suhrkamp.

Münch, S. (2016): Interpretative Policy-Analyse. Eine Einführung. Wiesbaden: Springer VS.

OECD (2011): How's Life? Measuring Well-being 2011. Paris: OECD.

Offe, C. (2006 [1972]): Strukturprobleme des kapitalistischen Staates. Veränderte Neuausgabe. Frankfurt/M.: Campus.

Pesendorfer, K./Leitner, F. (2010): Neue Zugänge zur Messung von Wohlstand und gesellschaftlichem Fortschritt. Wirtschaftspolitische Blätter, 4/2010, 533-544.

Pesendorfer, K./Eiffe, F./Wegscheider-Pichler, A. (2012): Wie geht's Österreich? Messung von Wohlstand und Fortschritt - Implementierung der SSF/ESS Empfehlungen. Wien: Statistik Austria.

Pierson, P. (2000): Increasing Returns, Path Dependence, and the Study of Politics. The American Political Science Review, 94 (2), 251-267.

Rein, M./Schön, D. (1993): Reframing Policy Discourse. In: Fischer, F./Forester, J. (Hg.): The Argumentative Turn in Policy Analysis and Planning. Durham: Duke University Press, 145-166.

Scheiblecker, M./Bock-Schappelwein, J./Sinabell, F. (2011): Ausgewählte Ergebnisse einer erweiterten Wohlstandsmessung im Ländervergleich. WIFO Monatsberichte, 11/2011, 713-726.

Schmelzer, M. (2016): The Hegemony of Growth. The OECD and the Making of the Economic Growth Paradigm. Cambridge: Cambridge University Press.

Schmidt, V.A. (2011): Speaking of change: why discourse is the key to the dynamics of policy transformation. Critical Policy Studies, 5 (2), 106-126.

Schneider, V./Janning, F. (2006): Politikfeldanalyse. Akteure, Diskurse und Netzwerke in der öffentlichen Politik. Wiesbaden: VS Verlag.

Sen, A. (2000): Ökonomie für den Menschen. Wege zu Gerechtigkeit und Solidarität in der Marktwirtschaft. München: Carl Hanser Verlag.

Skidelsky, R./Skidelsky, E. (2014): Wie viel ist genug? Vom Wachstumswahn zu einer Ökonomie des guten Lebens. München: Goldmann Verlag.

Statistik Austria (2015): Wie geht's Österreich? 2015 Indikatoren und Analysen. Wien: Statistik Austria.

Stiglitz, J./Sen, A./Fitoussi, J.P. (2009): Report by the Commission on the Measurement of Economic Performance and Social Progress. Paris.

SVR/CAE (Sachverständigenrat zur Begutachtung der gesamtwirtschaftlichen Entwicklung/Conseil d'Analyse Économique) (2010): Wirtschaftsleistung, Lebensqualität 
und Nachhaltigkeit: Ein umfassendes Indikatorensystem. Wiesbaden/Paris.

Thie, H. (2013): Rotes Grün. Pioniere und Prinzipien einer ökologischen Gesellschaft. Hamburg: VSA.

Whitby, A./Seaford, C./Berry, C. (2014): BRAINPOoL Project Final Report: Beyond GDP - From Measurement to Politics and Policy. London. 